\title{
Chronic Disruption of the Late Cholesterol Synthesis Leads to Female-Prevalent Liver Cancer
}

\author{
Kaja Blagotinšek Cokan ${ }^{1}$, Žiga Urlep ${ }^{1}$, Gregor Lorbek ${ }^{1}$, Madlen Matz-Soja ${ }^{2,+}{ }^{\circledR}$, Cene Skubic ${ }^{1}$, \\ Martina Perše ${ }^{3}{ }^{(1)}$, Jera Jeruc ${ }^{4}$, Peter Juvan ${ }^{1}$, Tadeja Režen ${ }^{1}$ ) and Damjana Rozman ${ }^{1, *}$ \\ 1 Centre for Functional Genomics and Bio-Chips, Institute of Biochemistry, Faculty of Medicine, \\ University of Ljubljana, SI-1000 Ljubljana, Slovenia; kaja.blagotinsek@mf.uni-lj.si (K.B.C.); \\ zigaurlep@gmail.com (Ž.U.); greg.lorbek@gmail.com (G.L.); cene.skubic@mf.uni-lj.si (C.S.); \\ peter.juvan@mf.uni-lj.si (P.J.); tadeja.rezen@mf.uni-lj.si (T.R.) \\ 2 Rudol-Schönheimer-Institute of Biochemistry, Divison of General Biochemistry, Faculty of Medicine, \\ University of Leipzig, 04103 Leipzig, Germany; madlen.matz@medizin.uni-leipzig.de \\ 3 Medical Experimental Centre, Institute of Pathology, Faculty of Medicine, University of Ljubljana, \\ SI-1000 Ljubljana, Slovenia; martina.perse@mf.uni-lj.si \\ 4 Institute of Pathology, Faculty of Medicine, University of Ljubljana, SI-1000 Ljubljana, Slovenia; \\ jera.jeruc@mf.uni-lj.si \\ * Correspondence: damjana.rozman@mf.uni-lj.si; Tel.: +386-1-543-7591 \\ + Current Address: Section of Hepatology, Clinic and Polyclinic for Gastroenterology, Hepatology, \\ Infectiology, Pneumology, University Clinic Leipzig, 04103 Leipzig, Germany.
}

Received: 14 September 2020; Accepted: 5 November 2020; Published: 9 November 2020

Simple Summary: Hepatocellular carcinoma is a disease with a variety of molecular triggers and is usually reported to prevail in males. However, after the menopause, the disease is also increasing in the female population. Herein, we discovered that chronic depletion of cholesterol synthesis due to the knock-out of the gene Cyp51 from this pathway leads to female prevalent hepatocarcinogenesis in aging mice. There is a high similarity between our mouse model and the situation in humans. Multiple deregulated pathways of hepatocarcinogenesis are shared. A female-dependent metabolic reprogramming leading to this type of liver cancer is exposed for the first time and reflects on deregulated cholesterol synthesis as the metabolic trigger. These data are of crucial importance. Despite the higher overall prevalence of hepatocellular carcinoma in males, we need tools and biomarkers to further stratify patients and offer better diagnosis and treatment options to both sexes.

\begin{abstract}
While the role of cholesterol in liver carcinogenesis remains controversial, hepatocellular carcinoma generally prevails in males. Herein, we uncover pathways of female-prevalent progression to hepatocellular carcinoma due to chronic repression of cholesterogenic lanosterol 14 $\alpha$-demethylase (CYP51) in hepatocytes. Tumors develop in knock-out mice after year one, with 2:1 prevalence in females. Metabolic and transcription factor networks were deduced from the liver transcriptome data, combined by sterol metabolite and blood parameter analyses, and interpreted with relevance to humans. Female knock-outs show increased plasma cholesterol and HDL, dampened lipid-related transcription factors FXR, $\mathrm{LXR} \alpha: \operatorname{RXR} \alpha$, and importantly, crosstalk between reduced LXR $\alpha$ and activated TGF- $\beta$ signalling, indicating a higher susceptibility to HCC in aging females. PI3K/Akt signalling and ECM-receptor interaction are common pathways that are disturbed by sex-specific altered genes. Additionally, transcription factors (SOX9)2 and PPAR $\alpha$ were recognized as important for female hepatocarcinogenesis, while overexpressed $C d 36$, a target of nuclear receptor RORC, is a new male-related regulator of ECM-receptor signalling in hepatocarcinogenesis. In conclusion, we uncover the sex-dependent metabolic reprogramming of cholesterol-related pathways that predispose for hepatocarcinogenesis in aging females. This is important in light of increased incidence of liver cancers in post-menopausal women.
\end{abstract}


Keywords: cholesterol biosynthesis; hepatocellular carcinoma; lanosterol 14 $\alpha$-demethylase (CYP51); sex dimorphism

\section{Introduction}

Metabolic abnormalities result in metabolic associated fatty liver disease (MAFLD), previously termed (NAFLD), which affects over $30 \%$ of the western population and has no approved drug therapies [1]. MAFLD can further develop into hepatocellular carcinoma (HCC) and both diseases are predicted to increase in the following decades [2], not only in men but also in women [3].

Among the lipid factors associated with progressive liver damage is also cholesterol. The cellular cholesterol concentrations are fine-tuned by a balance of synthesis, uptake, and efflux [4]. Genes of cholesterol synthesis differ in their susceptibility to loss of function mutations and can affect cell metabolism in different manners, from promoting cell survival towards manifesting in malignancies $[5,6]$. Linking cholesterol synthesis and liver disease remains controversial. Cholesterol synthesis was shown to support the growth of HCC lesions after depletion of fatty acid synthesis $[7,8]$, indicating crosstalk between fatty acid and cholesterol pathways in carcinogenesis. Overexpression of the squalene epoxidase enzyme of cholesterol synthesis was identified as a driver of NAFLD-induced HCC [9]. On the other hand, blocking cholesterol synthesis at the lanosterol $14 \alpha$-demethylase (CYP51) step leads to the prepubertal onset of liver injury with ductular reaction and fibrosis and a pronounced male prevalent liver dysfunction before puberty [10]. In young adults, the liver damage was more prominent among female mice due to diminished cholesterol esters and elevated sterol substrates [11].

The sex disparity in liver pathologies is far from conclusive. While some studies do not describe sex as a cofounding factor $[7,9,12,13]$, there is increasing evidence that after the menopause MAFLD occurs at a higher rate in women compared to men [14]. Female and male livers are metabolically distinct organs, which was demonstrated also in mathematical models of the liver with unique regulators of sex-specific metabolic outcomes [15]. A recent review concluded that clinical and epidemiological studies frequently fail to address sex differences appropriately [14].

This study aimed to address the long-term effect of disrupted liver cholesterol synthesis where the Cyp51 knock-out (KO) model of aging mice was applied in light of the relevance to humans. Surprisingly, the tumors developed spontaneously mainly in female aging mice. We identified novel metabolic pathways and transcription factors that can be linked to metabolism associated with female-prone hepatocarcinogenesis. We provide mechanistic insights into the metabolic reprogramming in this type of liver cancer, with special attention to that of females.

\section{Results}

\subsection{Long-Term Hepatocyte Deletion of Cyp51 from Cholesterol Synthesis Results in HCC}

We wanted to understand how the long-term depletion of cholesterol synthesis influences liver physiology. We expected that due to the damaged livers in young adulthood [16], the mice would have a shortened life span. In contrary to expectations, mice lived at least until 24 months (M). Their livers did not become cirrhotic but started to develop liver tumors between $12 \mathrm{M}$ and $24 \mathrm{M}$ of age. Hepatomegaly was more pronounced in females' KOs (Table S1). The histopathological examination of livers identified moderate to severe ductular reaction bridging the adjacent portal tracts, accompanied by mild inflammation (predominantly mononuclear cells; arrows in Figure 1A), and fibrosis. Importantly, fibrosis was more pronounced in females (Sirius red staining (SR); Figure 1B). Yellow pigment likely representing lipofuscin (arrow in Figure S1) was observed in areas of ductular reaction, sometimes surrounded by other hepatic cells, evaluated as macrophages (stars in Figure 1A). 


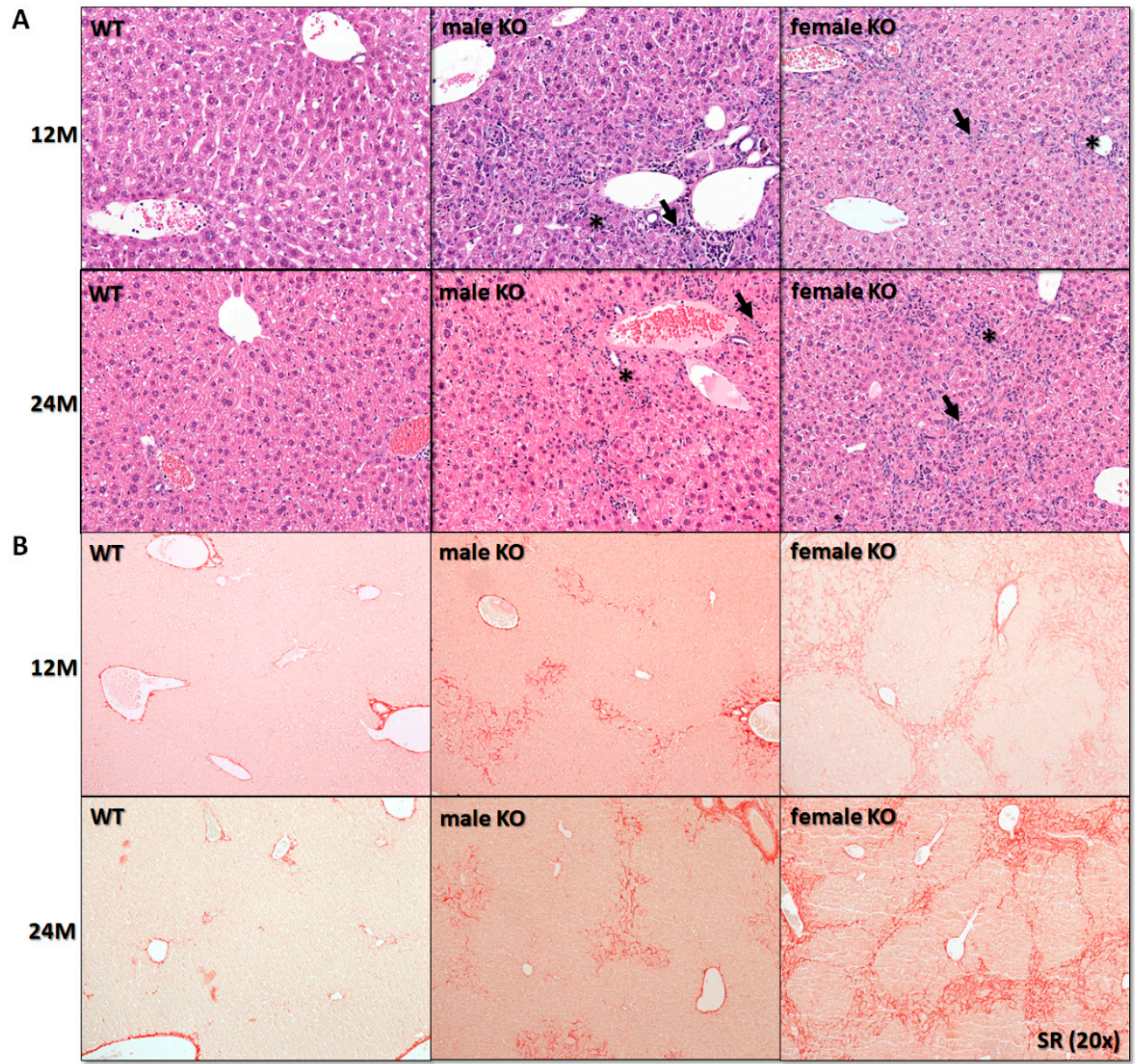

Figure 1. Blocking cholesterol synthesis due to depletion of Cyp51 resulted in histological alterations of the liver, most prominent in females. (A) Histological examination revealed ductular reaction $\left(^{*}\right)$ and inflammation $(\rightarrow)(H \& E$, original magnification $\times 200), N=5-10$. (B) Sirius red stain (SR, original magnification $\times 200$ ) showed more pronounced fibrosis in female transgenic mice, $\mathrm{N}=3-5$. M, month; $\mathrm{KO}, \mathrm{Cyp} 51 \mathrm{KO}$; WT, wild-type.

The incidence of tumors by sex end genotype is presented in Table S2. Liver tumors were observed initially in $12 \mathrm{M}$ female and $18 \mathrm{M}$ male $\mathrm{KO}$ mice (Figure 2Aa). They were defined as macroscopically visible nodules $(>1 \mathrm{~mm}$ ) of various colours. At $24 \mathrm{M}$, the incidence of tumors was $77.8 \%$ for females and $50 \%$ for males and were observed only in KO mice. In wild-types (WT), a single tumor was found in a 2-year male, histologically described as adenoma (Table S2). At 24M, the KO mice showed a decreased body weight compared to controls, significantly higher liver weight, and elevated relative liver weights. This was especially true for female mice with liver tumors (Figure S2 and Table S1). Tumors were histologically classified as eosinophilic/clear cell nodules, hepatocellular adenomas, or HCCs according to published criteria [17]. None of the tumors showed histological features of biliary tumor, e.g., biliary adenoma or cholangiocarcinoma.

Histologic evaluation is often sufficient for distinguishing the two types of primary hepatocellular tumors (HCC and cholangiocarcinoma), except in the case of poorly differentiated tumors. For further molecular analyses, we selected only tumors histologically classified as HCC, with features such as the absence of portal tracts and hepatic lobules, thickened hepatic plates, solid growth, focal tubular or pseudoglandular structures, focal necrosis, numerous apoptotic cells, and presence of cellular and nuclear polymorphism with brisk mitotic activity (Figure 2Ab,c). 


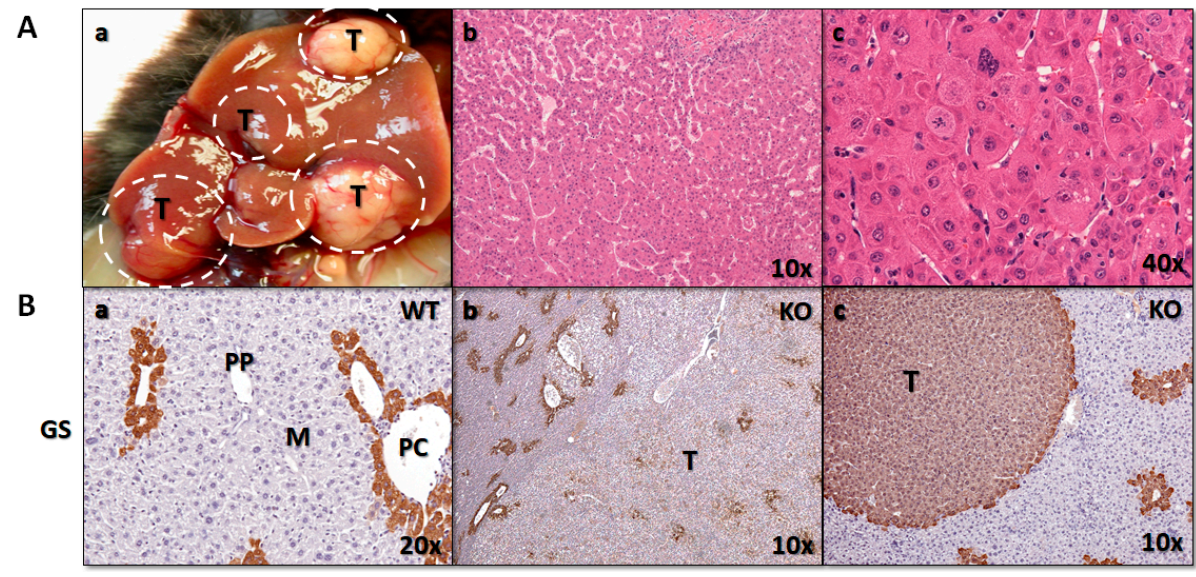

Figure 2. (A) Macroscopic and histological presentation of liver tumors. (a) Representative gross image of liver tumors in 24M KO mice. (b) Representative histologic image of HCC showing architectural abnormalities. (c) Higher magnification of (c), showing cellular and nuclear polymorphism with brisk mitotic activity. Hematoxylin and Eosin (H\&E), original magnification A, B $\times 100$, $\mathrm{C} \times 400$. (B) Immunohistochemical expression of GS marker of HCC in livers of WTs and KOs. Original magnification, (a) $\times 200$, (b) $\times 100,(\mathbf{c}) \times 400$. GS, glutamine synthetase; T, tumor; PP, periportal; PC, pericentral, M, midzonal; WT, wild-type; KO, Cyp51 KO; T, tumor.

Histologic evaluation is the gold standard in distinguishing the two most common primary hepatocellular tumors, namely hepatocellular carcinoma (HCC) and cholangiocarcinoma, except in the case of poorly differentiated tumors. Immunohistochemistry is a useful tool in determining the histogenesis of tumours. However, no single marker has been found that is completely specific for HCC. Among many different HCC markers, we decided for glutamine synthetase (GS), as it was recognized as the single best immunostain for identifying hepatocellular tumors in mice in a recent study [18]. As expected for normal liver tissue, positive GS staining was observed in pericentral hepatocytes (PC), but not in midzonal (M) or periportal (PP) hepatocytes (Figure 2Ba). Different staining patterns were observed in tumor tissue of Cyp51 KO mice, from scattered focal (Figure 2Bb), to diffuse staining of neoplastic hepatocytes (Figure 2Bc).

\subsection{Sex-Dependent Progression from Metabolism Associated Liver Disease towards HCC}

To learn about molecular changes based on long-term metabolic insult-the ablation of Cyp51 in hepatocytes - we assessed the hepatic expression of particular genes linked to cholesterol homeostasis during aging and measured multiple metabolites and markers in the blood of females and males. The results are presented graphically in Figure 3, with the most statistically significant changes between the groups (male, female, WT, Cyp51 KO) over all time points (12M, 18M, 24M) in Table S3. At the expression level, deregulation of cholesterol synthesis genes (Table S3) was, during aging, more pronounced in female livers (Figure 3A: Cyp51 KO-violet line, WT-black line) compared to those of males (Figure 3A: Cyp51 KO-green line, WT-blue line). The key regulatory gene of cholesterol synthesis $\mathrm{Hmgcr}$ is, over time, overexpressed in the KOs, particularly in females at $12 \mathrm{M}$. While at 12M Cyp51 is downregulated in the livers of Cyp51 KO, this is not the case anymore at older age. We were initially intrigued by this finding. However, the Cyp51 is knocked-out only in the hepatocytes and not in other cell types of the liver that seem to complement for the Cyp51 loss and increase its expression. If we look into the cholesterol synthesis gene expression in the tumors at $24 \mathrm{M}$ (Table S4), all genes except Cyp51 were upregulated, as expected for feedback regulation after the block in cholesterol synthesis, again significantly only in the female knock-outs. Investigation of bile acid (BA) synthesis pathway (Figure 3B, Table S3) showed the expression of Cyp8b1, Cyp7a1 and Cyp27a1 decreased in the KOs, with no significant sex differences. At $24 \mathrm{M}$ in tumors, Cyp7a1 is significantly downregulated in the female KOs (Table S4), indicating a drop in the classical pathway of BA synthesis. 
A
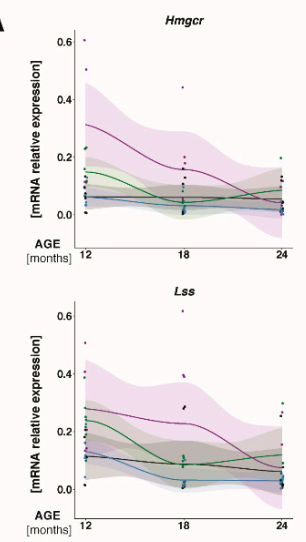

B

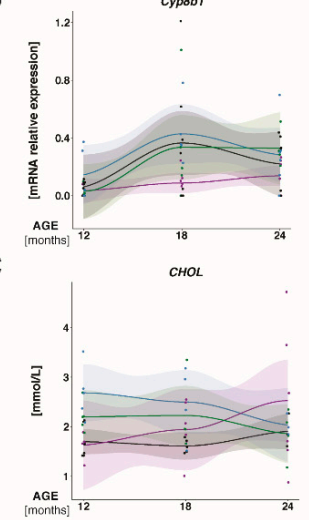

AL

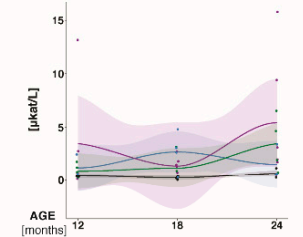

D
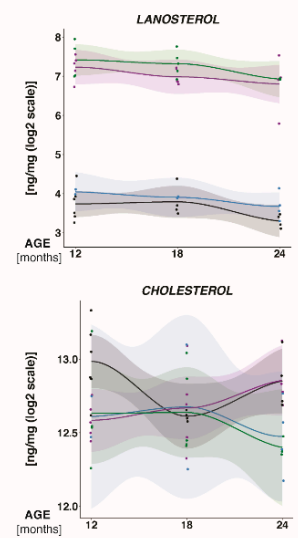
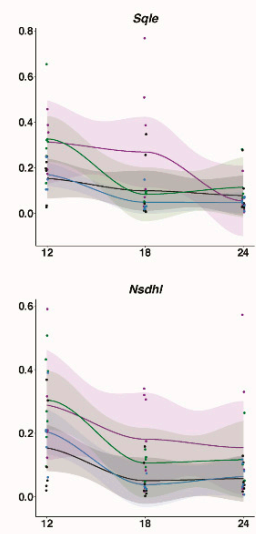

cyp7at

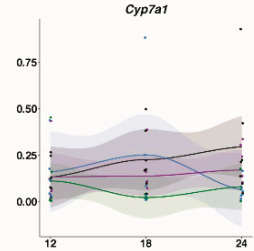

$H D L$

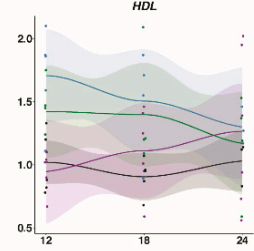

AST

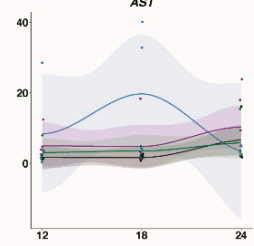

DIHYDROLANOSTEROL
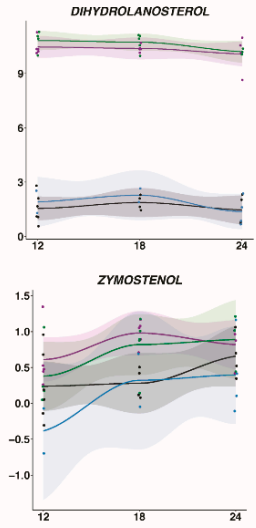
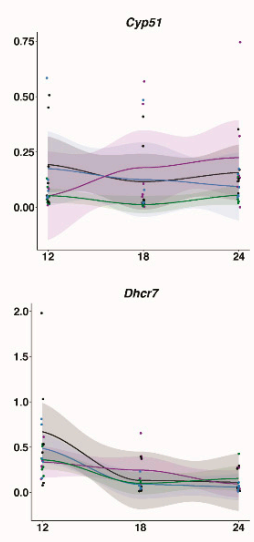

Cyp27at

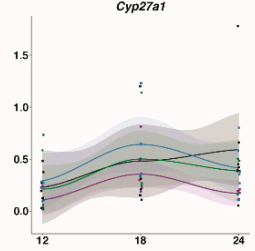

FFA
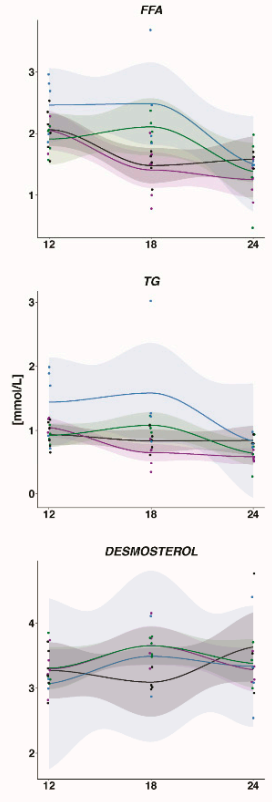

Sex.Genotype

= F.WT
= M.WT
F.KO

Figure 3. Adaptation to hepatocyte block in cholesterol synthesis during aging. Measurements were performed in $\mathrm{KO}$ and WT mice at $12 \mathrm{M}, 18 \mathrm{M}$ and $24 \mathrm{M}$ of age. Expression of hepatic genes from (A) from cholesterol synthesis (B) and bile acid synthesis. $\mathrm{N}=5$ mice per sex/genotype/age group. (C) Plasma concentration of biochemical parameters, $N=5-10$ mice per sex/genotype/age group. CHOL—cholesterol, HDL—high-density lipoproteins, FFA—-free fatty acids, ALT—alanine aminotransferase, AST-aspartate aminotransferase. (D) Liver concentration of sterol intermediates and cholesterol, $\mathrm{N}=4-6$ mice per sex/genotype/age group. Light color bands-95\% confidence interval. Dots-individual measurements. KO, Cyp51 KO; WT, Cyp51 WT, F = female; $\mathrm{M}=$ male. 
A major sex disparity was observed when monitoring the blood plasma biochemical parameters during aging. Total plasma cholesterol, free fatty acids (FFA), HDL (high-density lipoprotein cholesterol), and triglycerides (TG) showed opposite trends between sexes. Cholesterol and HDL were higher in $\mathrm{KO}$ females and were increasing by age, while they were lower in $\mathrm{KO}$ males compared to male controls, and decreasing with age. A significant increase in ALT in female Cyp51 KO mice is an additional indication of chronic hepatic injury (Figure 3C and Table S3), which is more prominent in females.

To quantify the primary molecular insults in the livers, we measured sterol intermediates of cholesterol synthesis together with cholesterol (Figure 3D and Table S3). In the KO mice with inactivated CYP51A1 enzyme, its substrates lanosterol and dihydrolanosterol were highly elevated ( 100 -fold and $\sim 500$-fold, respectively), proving disrupted cholesterol synthesis. Dihydrolanosterol was higher than lanosterol, indicating that 24-dehydrocholesterol reductase (DHCR24) enzyme functions normally. Downstream the pathway, desmosterol was not significantly different between Cyp51 KO and WT mice; however, zymostenol was higher in Cyp51 KO livers. Small or no changes between the sexes were observed in intermediate sterols. In contrast to that, sex represents a statistically significant parameter for liver cholesterol in the interaction analysis between sex and age. Similarly as for plasma cholesterol, hepatic cholesterol is also increased in female mice during ageing, while this was not the case for males (Figure 3C,D and Table S3).

\subsection{Transcriptome Analysis Identified Sex Differences in Gene Expression Linked to Hepatocarcinogenesis}

We used Affymetrix microarrays for gene expression profiling of mouse liver samples from $24 \mathrm{M}$ Cyp51 KO mice (24M KO) and controls (24M WT). We performed an analysis of liver tumors and the surrounding tissue that is responsible for tumorigenesis. To monitor the progression of tumorigenesis, data was compared to precancerous stages at 19 weeks of age (19W KO versus 19W WT) [16] and discussed in view of the early processes towards carcinogenesis (Tables S5-S7).

Hierarchical clustering of differentially expressed (DE) genes showed a clear difference between 24M WT and 24M KO mice for both sexes (Figure 4A). Importantly, a clear sex imbalance in the number of DE genes was observed (Figure 4B), with more genes (63.4\%) deregulated in the female livers, including Ctnnb1, Gsk3b, Nova1, Lmna, Gstp1, and Mup20 (Table S8). Among the DE genes deregulated only in males (11.6\%) were Trp53, Ezh2, Foxa1, and Rb1. In both sexes, a majority of DE genes were positively enriched (56\% in females and $68 \%$ in males) (Figure 4B). Using NetworkAnalyst [19], the functionally enriched networks of DE genes showed that positively enriched genes were mostly involved in pathways related to cancer and the extracellular matrix (ECM)-receptor interaction pathway, while genes from metabolic pathways were negatively enriched, especially among female KOs (Figure 4C).

Additionally, we selected the top 10 statistically sex-specific significant down- and up-regulated genes (Table S9), whose biological functions were examined. Lipid metabolism genes are among the most down-regulated, however, with some distinct differences in lipid related pathways in $24 \mathrm{M}$ $\mathrm{KO}$ females and $\mathrm{KO} 24 \mathrm{M}$ males. Most down-regulated genes in $\mathrm{KO}$ males are from metabolism of fatty acids, such as fatty acid elongation, and PPAR signalling. In the female KOs, we observed downregulation of steroid hormone biosynthesis and related metabolic pathways, bile secretion, and also fatty acid metabolism (arachidonic acids). Drug metabolism is also downregulated in KO females. Retinol and linoleic metabolism were among top 10 in both sexes.

Oppositely, top 10 up-regulated genes in male KO liver are mainly involved indifferent cancer pathways, PPAR signalling and ECM receptor interactions, while the female list includes other upregulated genes in PPAR signalling, Wnt signalling and diabetes pathways. 
A

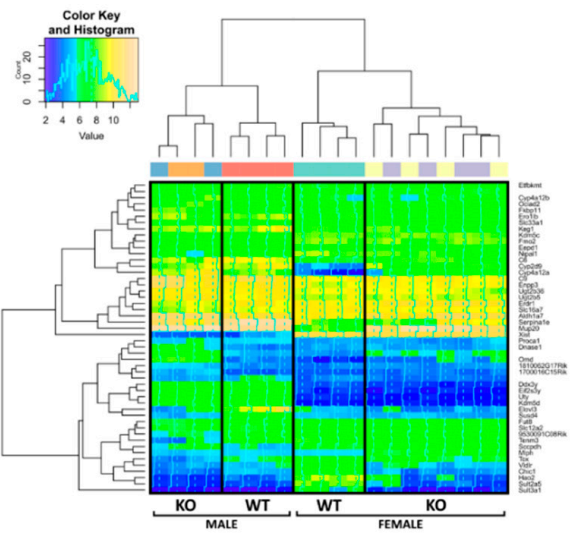

B

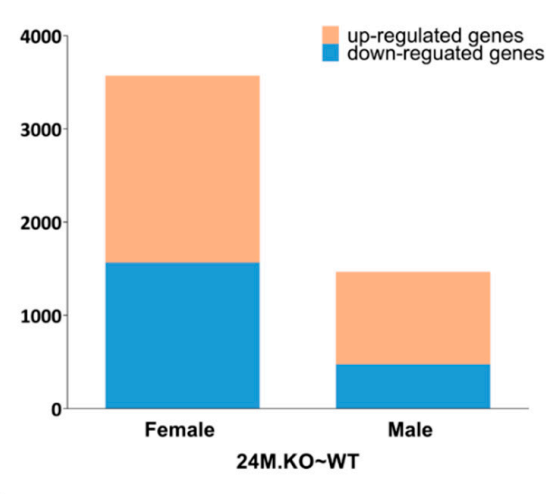

C

Molecular processes

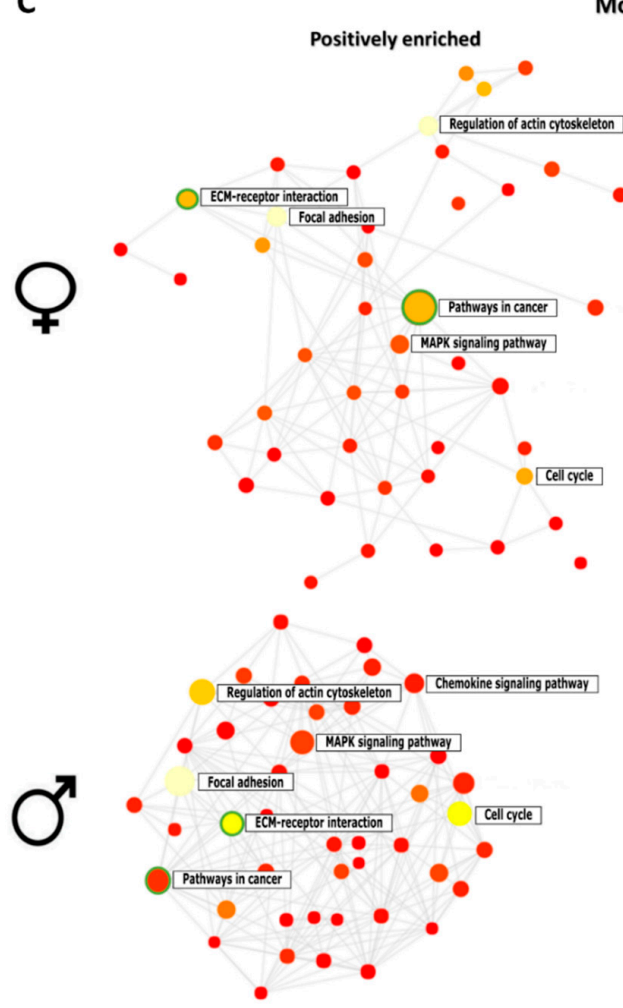

Negatively enriched

- Steroid hormone biosynthesis

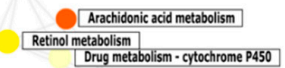

Drug metabolism - cytochrome P450 Drua metabolism - other enzymes Metabolism of xenobiotics by crtochrome P450
PPAR signaling pathway Fatty acid metabolism
Iryptophan metabolism Valine,leucine and issleucine degradation

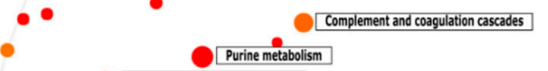

$\bullet$ Giycine, Serine and threonine metabolism

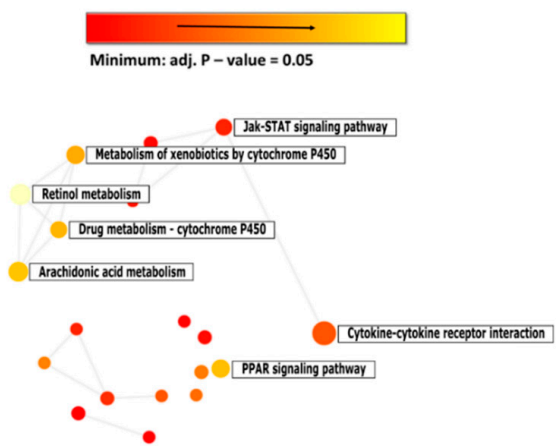

Figure 4. Target genes associated with blocked cholesterol synthesis. (A) Heat-map of top $50 \mathrm{DE}$ transcripts that showed significant differences in at least one comparison: hierarchical clustering distinguished between the genotypes and sex of $24 \mathrm{M}$ KO (24M.KO WT) mouse livers. (B) Stacked column plots with numbers of differentially up- and down-regulated sex-specific genes of 24M Cyp51 KO mice. (C) The functional enriched networks of up- and down-regulated DE genes in female and male 24M KO mice using NetworkAnalyst. The sizes of the nodes correspond to the number of DE genes within a particular molecular process. All pathway nodules are differentially expressed $(p<0.05)$, the gradation rising from red to yellow (most enriched) in the network of positively (left) and negatively (right) enriched molecular processes. Circles representing cancer pathways and ECM-receptor interaction are surrounded by green lines.

\subsection{Enriched KEGG Pathways and Transcription Factors (TFs) as Triggers of Female Prevalent Hepatocarcinogenesis}

From 323 KEGG pathways, we discovered 209 that were enriched in livers of precancerous 19W KO mice and 230 in 24M KO mice with tumors, compared to 24M WTs (Table 1 and Table S6). The enriched pathways include the activation of HCC, apoptosis, PI3K/Akt signalling, pathways in cancer, and proteoglycans in cancer. The ECM-receptor interaction pathway was observed to be the most positively enriched in 19W KO and 24M KO females and males (Table 1), which is in line with 
the histological findings. Chronic injury induces numerous molecular alterations in hepatocytes and causes a gradual accumulation of extracellular matrix components (ECM) that might lead to a complete architectural reconstruction of hepatocytes and tumor development.

Table 1. The most significantly enriched KEGG pathways in 19W KO (19W.KO WT) and 24M KO (24M.KO WT) mice. Parameters shown in bold are statistically significant. KEGG, Kyoto Encyclopaedia of Genes and Genomes; ${ }^{*} p<0.05,{ }^{* *} p<0.01,{ }^{* * *} p<0.001$.

\begin{tabular}{|c|c|c|c|c|c|c|c|c|}
\hline \multirow{3}{*}{ KEGG Pathway } & \multicolumn{4}{|c|}{ 19W.KO WT } & \multicolumn{4}{|c|}{ 24M.KO WT } \\
\hline & \multicolumn{2}{|c|}{ Females } & \multicolumn{2}{|c|}{ Males } & \multicolumn{2}{|c|}{ Females } & \multicolumn{2}{|c|}{ Males } \\
\hline & $\operatorname{LogFC}$ & $\begin{array}{c}\text { adj. } \\
p \text {-Value }\end{array}$ & $\operatorname{LogFC}$ & $\begin{array}{c}\text { adj. } \\
p \text {-Value }\end{array}$ & $\operatorname{LogFC}$ & $\begin{array}{c}\text { adj. } \\
p \text {-Value }\end{array}$ & $\operatorname{LogFC}$ & $\begin{array}{c}\text { adj. } \\
p \text {-Value }\end{array}$ \\
\hline Cell cycle & 1.13 & $0.01 *$ & 1.06 & 0.024 & 1.60 & $<0.001 * * *$ & 2.13 & $<0.001 * * *$ \\
\hline Pathways in cancer & 1.09 & $0.001 * *$ & 0.79 & $0.019 *$ & 1.89 & $<0.001 * * *$ & 1.84 & $<0.001 * * *$ \\
\hline ECM-receptor interaction & 1.30 & $0.005 * *$ & 1.50 & $0.005 * *$ & 1.82 & $<0.001 * * *$ & 1.85 & $<0.001 * * *$ \\
\hline Proteoglycans in cancer & 1.10 & $0.001 * *$ & 0.93 & $0.005 * *$ & 1.63 & $<0.001 * * *$ & 1.61 & $<0.001 * * *$ \\
\hline Apoptosis & 1.16 & $0.001 * *$ & 0.93 & $0.003 * *$ & 1.65 & $<0.001 * * *$ & 1.49 & $<0.001 * * *$ \\
\hline p53 signalling pathway & 0.67 & $0.011 *$ & 0.60 & $0.032 *$ & 1.26 & $<0.001 * * *$ & 1.33 & $<0.001 * * *$ \\
\hline MicroRNAs in cancer & 1.05 & $0.001 * *$ & 0.68 & 0.026 * & 0.87 & $<0.001 * * *$ & 0.95 & $<0.001 * * *$ \\
\hline Hepatocellular carcinoma & 0.67 & $0.001 * *$ & 0.32 & 0.131 & 0.95 & $<0.001 * * *$ & 0.72 & $0.006 * *$ \\
\hline Circadian entrainment & 0.21 & 0.123 & 0.13 & 0.387 & 0.42 & $0.020 *$ & 0.27 & 0.197 \\
\hline TGF- $\beta$ pathway & 0.61 & $0.002 * *$ & 0.38 & 0.051 & 0.45 & $0.007 * *$ & 0.14 & 0.427 \\
\hline Wnt signalling pathway & 0.29 & 0.051 & 0.10 & 0.588 & 0.32 & 0.018 * & 0.24 & 0.118 \\
\hline PI3K/Akt signalling pathway & 1.00 & $0.006 * *$ & 0.94 & $0.016 *$ & 1.61 & $<0.001 * * *$ & 1.51 & $<0.001 * * *$ \\
\hline Fatty acid degradation & -1.24 & $<0.001 * * *$ & -0.49 & 0.053 & -1.18 & $0.001 * *$ & -1.12 & $0.009 * *$ \\
\hline Metabolic pathways & -1.63 & $0.001 * *$ & -0.14 & 0.818 & -1.80 & $0.009 * *$ & -0.69 & 0.367 \\
\hline Peroxisome & -0.69 & $0.007 * *$ & -0.57 & $0.033 *$ & -1.82 & $<0.001 * * *$ & -1.20 & $0.018 *$ \\
\hline Primary bile acid biosynthesis & -1.76 & $<0.001 * * *$ & -0.60 & 0.103 & -1.29 & $0.002 * *$ & -1.40 & $0.004^{* *}$ \\
\hline $\begin{array}{l}\text { Biosynthesis of unsaturated } \\
\text { fatty acid }\end{array}$ & -0.47 & 0.063 & -0.05 & 0.874 & -0.87 & 0.018 * & -0.46 & 0.261 \\
\hline
\end{tabular}

We discovered multiple signalling pathways that describe the sex-dependent metabolic reprogramming in liver cancer. Among them, TGF- $\beta$ signalling was significantly elevated only in Cyp51 KO female livers, both at the precancerous stage and also later (Table 1). Additionally, the Wnt signalling and circadian entrainment pathways were activated only in the cancers of $\mathrm{KO}$ females (Table 1). We further explored whether these pathways could serve as triggers for female hepatocarcinogenesis in the Cyp51 KO model. The selected protein markers of the TGF- $\beta$ and Wnt signalling were evaluated by qPCR and IHC (Figures S3 and S4). The TGF- $\beta$ showed a positive reaction, particularly in female Cyp51 $\mathrm{KO}$ mice, indicating this is a good biomarker to follow progression towards HCC, particularly in females.

Among lipid metabolic pathways, we found fatty acid degradation and primary bile acid synthesis negatively enriched in KO females at $19 \mathrm{~W}$ and $24 \mathrm{M}$, while this was the case only for $24 \mathrm{M} \mathrm{KO}$ males (Table 1), suggesting that the metabolic reprogramming of fatty acid and BA metabolism is active earlier in female carcinogenesis.

Considering the general alteration of DE genes and KEGG pathways in hepatocarcinogenesis, we also investigated the transcriptional landscape by transcription factor (TF) enrichment analysis. This analysis indicated 321 enriched TFs in the precancerous 19W KO livers and 142 in tumor containing 24M KO livers, both in comparison to WT littermates (Table S7). The enriched TFs with specific functions are listed in Table 2. From the positively enriched transcriptional activity SP1, C-JUN, JUND, C-FOS, AP1 and C/EBP $\beta$ were enriched already in the precancerous stage in both sexes, while PPAR $\alpha$, (SOX9)2 and NFATC1 are positively enriched only in females, starting already at the precancerous stage. HIF1 $\alpha$ was also enriched in females in the precancerous stage, and together with SOX9, also in tumors of both sexes. The list of negatively enriched TFs is shorter. Downregulated CLOCK is characteristic for HCC, irrespective of the sex. HNF4 $\alpha$ and RORC are negatively enriched in females already at the precancerous stage and in both sexes in tumors. NR1B1 is the only TF downregulated in the precancerous male livers and later in tumors of both sexes. $\operatorname{LXR} \alpha: \operatorname{RX} R \alpha$ is a female-specific HCC marker. 
Table 2. The most significantly enriched TFs in 19W KO (19W.KO WT) and 24M KO (24M.KO WT). Parameters shown in bold are statistically significant. ${ }^{*} p<0.05,{ }^{* *} p<0.01,{ }^{* * *} p<0.001$; n.s. - not significant. SP-1: Specificity protein 1; AP1: Activator protein 1; C/EBP $\beta$ : DNA damage-inducible transcript 3; PPAR $\alpha$ : Peroxisome proliferator-activated receptor alpha; HIF1 $\alpha$ : Hypoxia-inducible factor 1-alpha; NFATC1: Nuclear factor of activated T-cells, cytoplasmic 1; CLOCK: Circadian locomotor output cycles kaput; LXR $\alpha$ : Liver X receptor alpha; RXR $\alpha$ : Retinoid X receptor alpha; HNF4 $\alpha$ : Hepatocyte nuclear factor 4 alpha; ROR $\alpha$ : RAR-related orphan receptor alpha, RORC: RAR-related orphan receptor gamma; NR1B1: Retinoic acid receptor alpha.

\begin{tabular}{|c|c|c|c|c|c|c|c|c|}
\hline \multirow{3}{*}{$\begin{array}{l}\text { Transcription } \\
\text { Factor }\end{array}$} & \multicolumn{4}{|c|}{ 19W.KO WT } & \multicolumn{4}{|c|}{ 24M.KO WT } \\
\hline & \multicolumn{2}{|c|}{ Females } & \multicolumn{2}{|c|}{ Males } & \multicolumn{2}{|c|}{ Females } & \multicolumn{2}{|c|}{ Males } \\
\hline & $\log F C$ & $\underset{p \text {-Value }}{\text { adj. }}$ & $\log F C$ & $\underset{p \text {-Value }}{\text { adj. }}$ & $\log F C$ & $\underset{p \text {-Value }}{\text { adj. }}$ & $\operatorname{LogFC}$ & $\begin{array}{c}\text { adj. } \\
p \text {-Value }\end{array}$ \\
\hline SP1 & 0.81 & $0.002 * *$ & 0.85 & $0.001 * *$ & 1.47 & $<0.001 * * *$ & 1.49 & $<0.001 * * *$ \\
\hline C-JUN & 0.93 & $0.001 * *$ & 0.75 & $0.005 * *$ & 1.42 & $<0.001 * * *$ & 1.50 & $<0.001 * * *$ \\
\hline JUND & 0.92 & $0.002 * *$ & 0.72 & 0.013 * & 1.05 & $<0.001 * * *$ & 0.91 & $<0.001 * * *$ \\
\hline C-FOS & 1.20 & $<0.001 * * *$ & 0.98 & $<0.001 * * *$ & 1.47 & $<0.001 * * *$ & 1.33 & $<0.001 * * *$ \\
\hline AP1 & 1.00 & $<0.001 * * *$ & 0.66 & $0.003 * *$ & 1.17 & $<0.001 * * *$ & 0.88 & $<0.001 * * *$ \\
\hline $\mathrm{C} / \mathrm{EBP} \beta$ & 0.52 & $0.010 *$ & 0.76 & $<0.001 * * *$ & 0.91 & $<0.001 * * *$ & 0.87 & $0.004 * *$ \\
\hline $\operatorname{PPAR} \alpha$ & 0.51 & $0.04 *$ & 0.06 & 0.83 & 0.60 & $0.001^{* *}$ & 0.22 & 0.27 \\
\hline SOX9 & 0.34 & 0.082 & 0.30 & 0.125 & 0.90 & $<0.001 * * *$ & 0.63 & $0.001 * *$ \\
\hline$(\mathrm{SOX} 9) 2$ & 0.46 & 0.012 * & 0.33 & 0.057 & 0.67 & $0.005 * *$ & 0.50 & 0.062 \\
\hline $\mathrm{HIF} 1 \alpha$ & 0.33 & 0.013 * & 0.23 & 0.070 & 0.43 & $0.008 * *$ & 0.58 & $0.004 * *$ \\
\hline NFATC1 & 0.34 & 0.042 * & 0.18 & 0.284 & 0.36 & 0.035 * & 0.29 & 0.156 \\
\hline CLOCK & -0.24 & 0.035 & -0.17 & 0.122 & -0.26 & 0.031 * & -0.41 & $0.009^{* *}$ \\
\hline $\mathrm{LXR} \alpha: \operatorname{RXR} \alpha$ & n.s. & n.s. & n.s. & n.s. & -0.56 & $0.043 *$ & -0.19 & 0.602 \\
\hline $\mathrm{HNF} 4 \alpha$ & -0.73 & $0.007^{* *}$ & -0.27 & 0.290 & -1.60 & $<0.001 * * *$ & -1.17 & $0.019 *$ \\
\hline $\mathrm{ROR} \alpha$ & -0.72 & $<0.001 * * *$ & -0.20 & 0.256 & n.s. & n.s. & n.s. & n.s. \\
\hline RORC & -0.35 & 0.028 * & -0.11 & 0.474 & -1.68 & $<0.001 * * *$ & -2.10 & $<0.001 * * *$ \\
\hline NR1B1 & -0.21 & 0.087 & -0.35 & $0.007^{* *}$ & -0.80 & $<0.001 * * *$ & -0.59 & $<0.001 * * *$ \\
\hline
\end{tabular}

When we considered the top-10 sex-specific enriched TFs based on the fold-change (Table S10), additional information was gathered. PPAR $\alpha$ and the oncogenic isoform (SOX9)2, which were exposed previously (Table 2), are among top 10 positively enriched TFs by fold-change in females. FXR is, by fold-change, the most downregulated female-specific TF in HCC that was not exposed in the overall TF deregulation of Table 2. LXR $\alpha: R X R \alpha$ were, again, among the most negatively enriched TFs in female tumors (Table S10). SOX9 is a part of the Wnt signalling and is expressed in the progenitor/precursor cells during hepatocyte regeneration following liver injury [20]. Expression of heterodimer complex LXR $\alpha: R X R \alpha$ correlates with the amount of the retinoic acid and cholesterol derivates [21], which is line with diminished activity in $\mathrm{KO}$ female mouse livers. It was shown that chronic administration of PPAR $\alpha$ agonists leads to liver tumor development in mice [22]. However, it is still unclear how this receptor changes in the human liver cancer tissue. An investigation of PPAR $\alpha$ mRNA expression in human HCC indicated an increased mRNA expression of PPAR $\alpha$ and its target genes. These results point out the possible association of activated PPAR $\alpha$ with human carcinogenesis [23]. NFATC1, which was not on the list of sex-specific top $10 \mathrm{TFs}$, was also indicated as female-specific TF (Table 2). The NFATC1 with calcineurin overexpression plays an important role in the proliferation of HCC [24]. NFATC1 transcription can be activated by AP1 (including FOS-JUN), the activation of which has been already described in T cells [25].

RORC (ROR $\gamma$ ) was one of the TFs in our focus since sterols after lanosterol are ligands of this transcription factor [26]. Downregulation of RORC signalling is thus important due to lowered intermediate sterols upon the Cyp51 block.

To validate whether downregulation of RORC has effects in hepatocarcinogenesis, we evaluated the expression of downstream genes regulated by this transcription factor. We found a majority of RORC target genes decreased in Cyp51 KO mice (Table S11), since RORC serves as their activator. However, the RORC target Cd36 was upregulated since RORC is known to repress this gene [10]. The RORC target G6pc, which is involved in the regulation of the PI3K/Akt signalling pathway, 
was negatively enriched in female $\mathrm{KO}$ mice, representing a part of the female metabolic reprogramming (Table S11).

A summary of sex-dependent reprogramming of metabolic pathways and corresponding transcription factors is shown in Figure 5. Briefly, Figure 5 indicates connections between signalling pathways, TFs and cellular processes, which were deregulated in both sexes (top half of figure) and specific for female KOs (bottom half of figure), leading to the inhibition of basal metabolism and an increased possibility for tumor development.

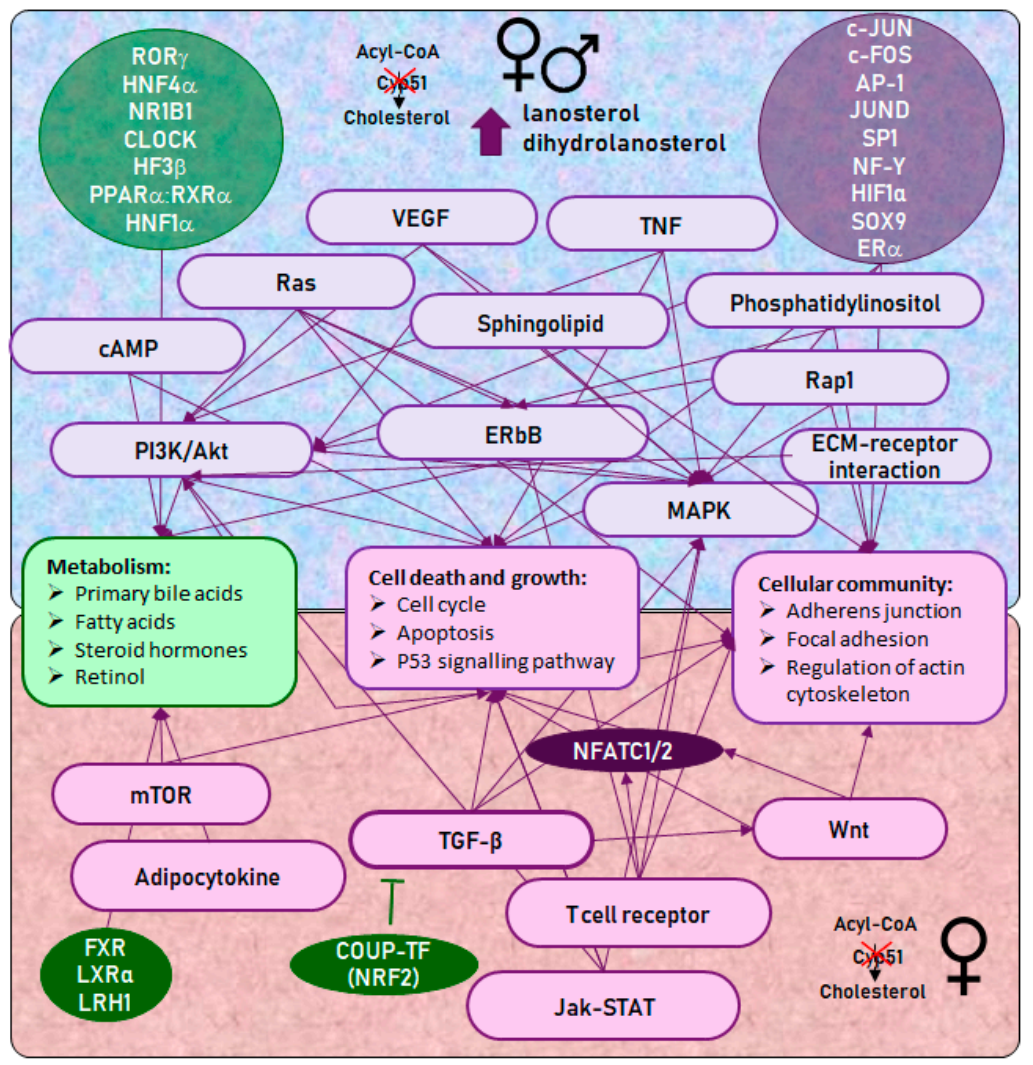

Figure 5. Hepatocyte-specific Cyp51 KO resulted in the deregulation of multiple signalling pathways and TFs, leading to the development of female-prevalent liver cancer. Arrows indicate connections between KEGG signalling pathways, TFs, and cellular processes. KEGG pathways and TFs are violet if positively enriched and green if negatively enriched at 24M. Top half-selected KEGG signalling pathways and TFs that were deregulated in females and males, leading to the inhibition of basic metabolism and an increase in processes of cell death and growth, and cellular communication. MAPK and PI3K/Akt signalling pathways have a central role and are regulated by other signalling pathways and TFs, all directly or indirectly contributing to carcinogenesis. Bottom half-female-specific KEGG signalling pathways and TFs. TF NFATC1/2 is central to the female-specific phenotype and is connected by the TGF- $\beta$, mTOR and Wnt signalling pathways, positively enriched only in female Cyp51 KO livers.

The TF networks were visualized with STRING [27] to determine their interactions. Different colours of nodes represent biological processes in which enriched TFs of Cyp51 KO mice are involved. Surprisingly, SP1 and JUND deregulated in precancerous and tumor livers of both sexes represent nodules with most protein-protein associations linked to hepatocarcinogenesis (Figure S5). 
2.5. Metabolic and Transcriptional Changes after Disrupted Cyp51 from Cholesterol Synthesis Align with Hepatocarcinogenesis in Humans

We performed a comparative analysis of sex-dependent metabolic adaptations upon inactivation of CYP51A1 in the hepatocytes in light of the human literature data. Figure 6 represents the proposed carcinogenesis mechanism in our mouse model with exposed signalling, their specific sex-dependent targets and possible transcription regulators that might offer clues for expanding the understanding of the human sex-dependent HCC carcinogenesis. In the mouse model, the chronic metabolic insult of disrupted cholesterol synthesis (accumulation of hepatic sterols lanosterol and dihydrolanosterol) resulted in a variety of sex-specific metabolic adaptations. The hepatic cholesterol was increased in $\mathrm{KO}$ females and decreased in $\mathrm{KO}$ males. This observation serves as one of the important features in cancer cells. As fast proliferating cells, cancer cells require high levels of cholesterol for membrane biogenesis and other functional needs. For example, the cholesterol-derived oncometabolite 6-oxo-cholestan- $3 \beta, 5 \alpha$-diol was enriched in patients with breast cancer and subsequently promotes tumor growth [6].

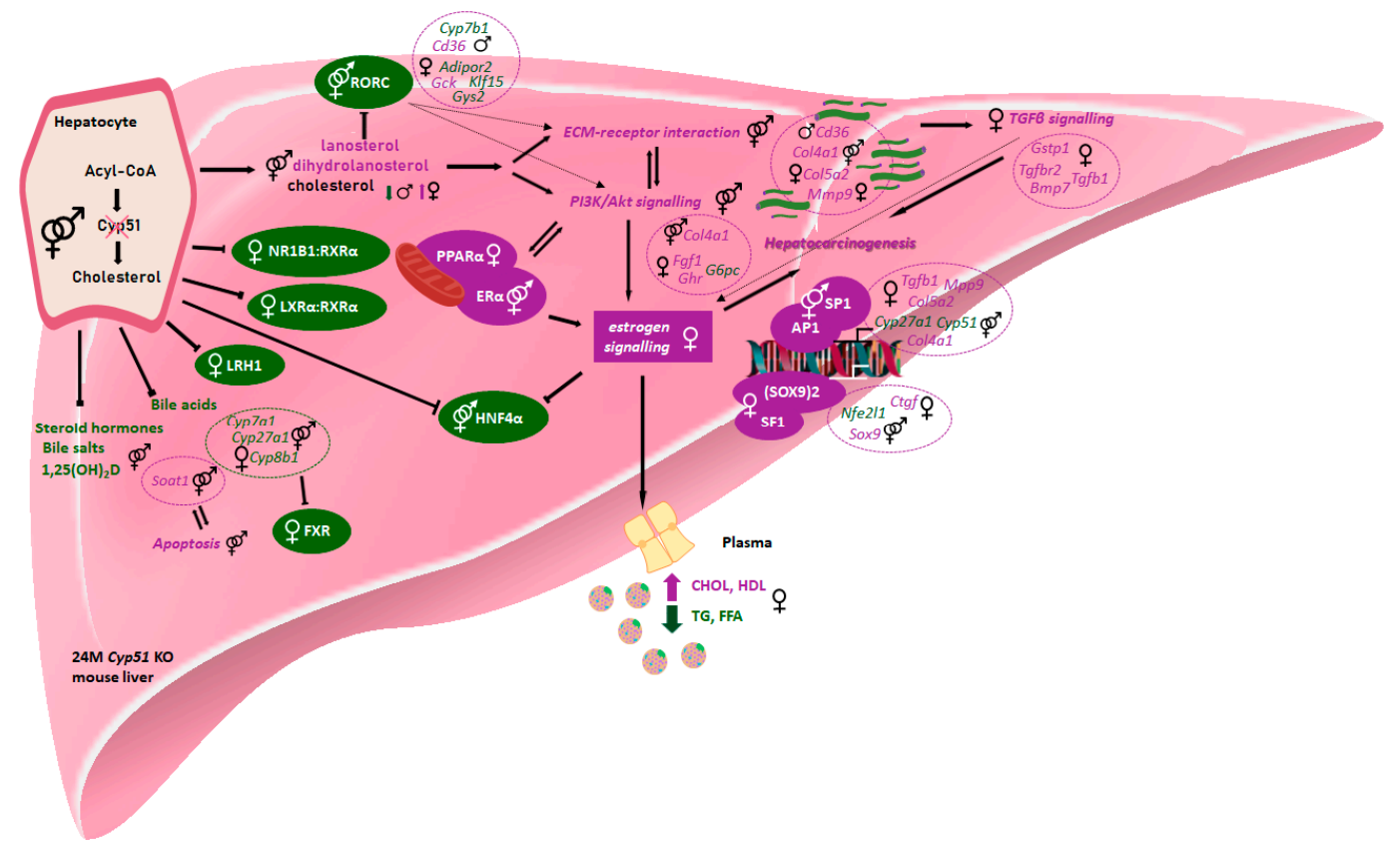

Figure 6. Sex-dependent metabolic and transcriptional changes after disrupted Cyp51 from cholesterol synthesis align with hepatocarcinogenesis in humans. Arrows indicate active connections between enriched genes, KEGG pathways, and TFs. Blocked arrows represent repression. Hatched arrows represent possible transcription regulation of the selected pathway or connection between selected pathways in KO mice. Metabolites, DE genes, KEGG pathways, and TFs are violet if positively enriched and green if negatively enriched. Icons were used from the Reactome icon library. All selected mouse signalling and metabolic pathways and TFs were aligned with human literature data.

As shown in Figure 6, disturbance of cholesterol synthesis leads to the repression of selected nuclear receptors (FXR, LXR $\alpha: R X R, L R H 1$ and NR1B1:RXR $\alpha$ ) in female KO hepatocytes, which are increasingly appreciated targets in the prevention of inflammation and fibrosis [6,28]. The positive enrichment of PPAR $\alpha$, observed only in females, was also confirmed in humans with NAFLD [21], but its role in HCC patients remains unclear [29]. FXR negative enrichment and downregulation is also in line with the human HCC progression. Negative enrichment of FXR in females and downregulation of its target gene $\mathrm{Ndrg} 2$ is in line with the human data in patients and confirmed tumor suppressor role of FXR and NDRG2 in human cell lines [30]. 
Aligned with the latest human literature, metabolic and transcriptional changes of disturbed cholesterol metabolism in females pinpoints on a new sex-dependent mechanism, which has not been revealed so far. We proposed that a higher incidence of HCC in females results from the crosstalk of downregulated transcriptional activity of $\operatorname{LXR} \alpha$ and activation of TGF $\beta$ signalling. Positive expression of TGF- $\beta$ signalling targets Gstp1, Tgfrb1, Tgfrb2 and Bmp7 was identified only in female $\mathrm{KO}$ mice. Among several human malignancies, TGF- $\beta$ in humans regulates the progression of female HCC through the promotion of PI3K/Akt signalling [31] and ECM-receptor interaction [32]. PI3K/Akt signalling and ECM-receptor interaction are common pathways that are disturbed by altered gene expressions in human cancers $[33,34]$. In our mouse model, both pathways were activated, but through different positively enriched genes in females (Col5a2 and Mmp9) compared to males (Cd36). In the context of lipid homeostasis, Raichur S et al. showed the opposite expression patterns, where repressed RORC activity induces expression of Cd36. In both animal and human studies, increased $C d 36$ expression in metabolically active tissue has been implicated in the regulation of fatty acid metabolism and lipid accumulation $[35,36]$. In HCC patients, the number of fatty acid responsive TFs, including HIF1 $\alpha$ and FOS, can transcriptionally upregulate $C d 36$, which is closely associated with induction of epithelial-mesenchymal transition (EMT) [37].

Moreover, Gck, which is known as a one of RORC targets, was downregulated in Rorc ${ }^{-/-}$mice [38], while in female Cyp51 KO livers, it was upregulated. The identity of all TFs regulating Gck promoter activity has not been fully elucidated. One of the possible co-regulations of the liver Gck expression may be through the insulin, which can increase Gck mRNA via PI3K/Akt signalling, regulating the $\mathrm{HIF1} \alpha[39]$ that were both found to be activated in $\mathrm{KO}$ mice.

In humans, the expression of SOX9 correlates with the severity of liver fibrosis patients [40] and correlates with the progression to cirrhosis and HCC [41]. In our case, the activated TF (SOX9)2, and its targets Ctgf and Nfe2l1 (Table S11), were deregulated only in female KOs.

\section{Discussion}

The hallmark of cancer cells is metabolic reprogramming, causing new cellular demands in selective survival and growth [42]. Cholesterol is a precursor of steroid hormones and bile acids and an essential component of cellular membranes. Its circulating levels are under healthy homeostatic conditions regulated by a balance between cellular cholesterol synthesis, dietary intake, and removal of excess cholesterol [43].

CYP51A1 is a gene from the late (post-squalene) part of the pathway [44]. The disruption of this gene in hepatocytes starts with morphological alterations, such as ductular reaction accompanied by mild inflammation. We show herein that this can end with malignant liver tumors in ageing mice that are first observed at $12 \mathrm{M}$ in females and $18 \mathrm{M}$ in males, with the highest incidence in the $24 \mathrm{M}$ female Cyp51 KO mice. In addition, humans show sex-dependent differences in liver pathologies. Women more commonly present with acute liver failure, benign liver lesions, autoimmune hepatitis, and toxin-mediated hepatotoxicity. Oppositely, malignant liver tumors and viral hepatitis are less commonly observed in women [45]. However, non-alcoholic steatohepatitis (NASH), as a more serious form of MALD, more commonly progresses to HCC in women [46]. Generally, women are less prone to developing HCC than men (due to hormone-dependent resistance), while after the menopause a sharp increase in HCC incidence is witnessed [47].

Based on our data, we propose that the repression of CYP51A1 enzyme can contribute to HCC development. In humans, whole-body deletion of CYP51A1 is not reported, since this gene is essential and is embryonically lethal in the mouse [48]. Only two studies are reporting the CYP51A1 deleterious mutations, which resulted in infant liver failure and death [49,50]. Numerous polymorphisms in CYP51A1 have been associated with different phenotypes in humans [51], but the consequences of CYP51A1 heterozygosity in human hepatocarcinogenesis need yet to be established. CYP51A1 has a low mutation rate, lower than other cholesterol synthesis genes [52]. The CYP51A1 rs37417517 variant was found to be associated with the female-specific breast cancer and variant rs229188 with female genetics $[53,54]$. 
Additionally, the COSMIC database revealed the presence of somatic CYP51A1 point mutations in tumor samples, some also with a predicted deleterious effect on activity (Tables S12 and S13).

The synthesis of cholesterol is a housekeeping pathway, which includes more than 100 genes associated with cholesterol synthesis and its regulation [55]. The block in cholesterogenic Cyp51 in KO mice is in our case specific in hepatocytes. Thus, about $40 \%$ of Cyp51 gDNA remained unchanged in the livers of $\mathrm{KO}$ mice, which lead to an approximally $60 \%$ decrease in mRNA and an $80 \%$ decrease in the CYP51 protein. Lorbek et al. indicated the small foci of stained periportal hepatocytes that potentially originated from the oval cell compartment by immunohistochemical staining of CYP51. Thus, the high intensity of ductular proliferation in ageing mice may explain an increase in Cyp51 mRNA in female and male KO livers from 18M onward [16]. Oppositely, the drop in expression of other genes of cholesterol synthesis could be a result of hepatic inadequacy due to chronic liver disease, which was also shown in fibrotic patients [56].

The latest studies indicate that post-squalene intermediates of cholesterol synthesis are also signalling molecules. Inhibiting different enzymes from the pathway results in the accumulation of sterol intermediates with different cellular effects. Sterol products can activate RORC signalling [26], promote oligodendrocyte formation [57], or activate LXR $\alpha$ and inhibit EGFR signalling [58]. Inhibitors of lanosterol synthase were identified as potential anticancer drug targets [59] while the accumulation of lanosterol can cause degradation of 3-hydroxy-3-methyl-glutaryl-coenzyme A reductase (HMGCR) [60]. Our sterol measurements show significant accumulation of CYP51A1 substrates lanosterol and dihydrolanosterol, with no sex differences. We observed lower amounts of desmosterol and cholesterol in $\mathrm{KO}$ livers. The presence of desmosterol in the liver may be explained by compensatory effects on lower levels of DHCR24, as it was shown in the brain [61]. The liver cholesterol may be obtained from the diet, to compensate the defected de novo synthesis of cholesterol [62]. The increase in cholesterol in female KO livers may arise due to the cholesterol uptake from plasma, since cholesterol is required for tumor cell proliferation and growth $[56,63]$.

The main difference between the genotypes is likely in the accumulation of sterol substrates rather than a lack of downstream sterols, while the difference between the sexes is shown by the sex-dependent transcriptome response to sterol imbalance. Due to the lack of sterols between lanosterol and zymosterol, which are proposed RORC natural ligands, we observe at $24 \mathrm{M}$ the repression of the RORC signalling pathway in both sexes. The RORC-dependent mechanisms in cancerogenesis were recently described. Oh et al. reported that RORC expression is decreased in basal-like subtype cancers and inversely correlated with histological grade and cancer drivers in breast cancer cohorts [64]. In breast cancer cell lines, RORC was shown to repress TGF- $\beta$ /EMT-signalling and cancerogenic pathways, which indicates that, also in Cyp51 KO mice, decreased activity of RORC could contribute to increased TGF- $\beta /$ EMT signalling. There is no data yet that would link SOX9 expression to sterols. (SOX9)2 is upregulated, particularly in KO females, and further research is needed to evaluate whether this is a direct effect of accumulating lanosterol and dihydrolanosterol.

Injured hepatocytes in Cyp51 KO mice produce different ECMs, exemplified by the enrichment of laminin and collagen genes (Col4a1, Lamc1, etc.) and a strongly positively enriched ECM-receptor interaction pathway in hepatocarcinogenesis of both sexes. In some way, these alterations could contribute to microenvironmental reorganization and induction of EMT that positively influences tumor progression [32]. A higher rate of cancer progression in female Cyp51 KO mice could be explained by the female-specific interplay of positively enriched TGF- $\beta$ signalling. Activated TGF- $\beta$ represents a central regulator of chronic liver disease and could contribute to the progression of all disease stages, both in animal models and in humans [65]. Interestingly, important crosstalk between TGF- $\beta$ and Cyp51 gene expression has been shown in the Tgf- $\beta \mathrm{fl} / \mathrm{fl} ; \mathrm{Wnt}-\mathrm{Cre}$ mouse model [66].

Furthermore, the results of our analysis identified the key transcriptional regulators above the differentially expressed molecular pathways: positively enriched SOX9, SP1, and negatively enriched RORC. SOX9 is an important candidate for the cancer marker required for activation of TGF- $\beta /$ Smad signalling [67]. SOX9 expression, especially its isoform 2 ((SOX9)2), can predict liver 
disease progression [40], but was so far not described as being associated more with the female type of carcinogenesis. Similarly to SOX9, the tumor progression driver SP1 is also a key factor for the induction of TGF- $\beta$ during inflammation [68] and its overexpression in Cyp51 knock-outs relates to EMT. The impact of higher disease progression in females is reflected also in the interplay of transcription regulator SMAD3 on TGF- $\beta$ signalling [69].

Increased plasma total cholesterol and HDL, and decreased plasma TG and FFA, are observed only in female KO mice. This is a possible effect of induced sex hormone-binding globulin (SHBG) by specific targets of an estrogen signalling pathway [70]. SHBG positively correlates with HDL and negatively with TGs and has been already associated with different cancers in ageing humans [71,72]. Supporting other hepatocarcinogenesis studies [73,74], the outcome of altered cholesterol and BA homeostasis was a chronic liver injury in our Cyp51 KO mouse model with widespread fibrosis, inflammation, and malignant transformation due to toxic sterol accumulation. Additionally, sterol accumulation, in our case the consequence of inhibition of CYP51A1 [16], could be a cause of the observed widespread increase in hepatic progenitors (also known as a ductular reaction) that may also differentiate into mature hepatocyte-like cells with oncogenic potential [75].

\section{Materials and Methods}

Detailed materials and methods are described in Appendix A. The experimental design of the study is shown in Figure A1.

\subsection{Animal Study and Samples Collection}

The generation of homozygous hepatocyte-specific Cyp51 knock-out (Cyp51 KO) mice has been reported previously $[10,16]$. Animals were bred under a $12 \mathrm{~h}$ light and dark cycle $(7: 00 \mathrm{a} . \mathrm{m}$. until 7:00 p.m. light), at $22 \pm 1{ }^{\circ} \mathrm{C}$, humidity $55 \pm 10 \%$, on standard rodent chow (diet 1324, Altromin, Lage, Germany) and acidified tap water $(\mathrm{pH}=3)$ ad libitum. They were housed in groups of $3-5$ mice per cage. The results of routine health microbiological monitoring are shown in Table A1. Mice were sacrificed at different ages $(12 \mathrm{M}, 18 \mathrm{M}$ and $24 \mathrm{M}$ ) with cervical dislocation between 11 and $13 \mathrm{~h}$ (to minimize the circadian influence), after a $4-6 \mathrm{~h}$ of fasting (food withdrawal at $7 \mathrm{~h}$ a.m.). Blood was taken immediately after cervical dislocation from the right ventricle, collected into heparin-coated Vacuette MiniCollect ${ }^{\circledR} 1 \mathrm{~mL}$ Plasma Tubes (Greiner Bioone, Frickenhausen, Germany) and centrifuged for $4{ }^{\circ} \mathrm{C}, 3000 \times g$ for $15 \mathrm{~min}$. Mice were weighed, organs (liver, kidney, spleen, heart, gonads) removed and patho-morphologically examined. For histology, the left lateral lobus was fixed in $4 \%$ formalin and embedded in paraffin for further histological analysis. Other parts of livers were cut into thin slices and snap-frozen in liquid nitrogen. The material was freshly frozen and stored at $-80{ }^{\circ} \mathrm{C}$ for subsequent analysis.

\subsection{Ethics Statement}

Mouse experiments were approved by the Veterinary Administration of the Republic of Slovenia (license numbers 34401-31/2011/4 and 34401-52/2012/3) and were conducted in agreement with the National Institute of Health guidelines for work with laboratory animals, national legislation and Directive 2010/63/EU on the protection of animals used for scientific purposes.

\subsection{Histological Analysis}

For standard histological analyses, formalin-fixed paraffin-embedded tissue was used, sectioned to 4-5 $\mu \mathrm{m}$ and deparaffinized at $70{ }^{\circ} \mathrm{C}$ for $10 \mathrm{~min}$ and washed in two changes of Xylene, $100 \%$ ethanol, $95 \%$ ethanol, 70\% ethanol and twice in acidified water. Hematoxylin and Eosin (H\&E) stained liver sections of each mouse were scored for size, shape, and polymorphism of the hepatocytes, granulation of cytoplasm, presence of portal and parenchymal inflammation, ductular reaction, cholestasis, and presence of any type of nodule. The intensity of inflammation and ductular reaction was graded as: 0 -normal, +- mild, ++- moderate and +++- severe. The extent of ductular 
reaction was assigned: $\mathrm{p}$-proliferation around portal tracts, $\mathrm{p}-\mathrm{mz}$ - proliferation extending from one portal tract to the middle zone, and p-p-bile ducts were extending from one portal field to another (bridging). For evaluation of liver fibrosis, Sirius red (SR) staining was performed by incubating deparaffinized sections in $0.1 \%$ Sirius Red solution for 1 h (SR; $0.1 \%$ direct red $80,1.2 \%$ picric acid in water), briefly destaining in diluted acetic acid, dehydrating in 70\% ethanol, 95\% ethanol, 100\% ethanol and twice in Xylene and fixed with Roti Histokitt II (Carl Roth GmbH + Co. KG, Karlsruhe, Germany). Fibrosis in SR stained liver samples were graded: 0 as absent, + mild periportal, ++ moderate, and +++ as very strong fibrosis with bridging. Tumors, identified as macroscopically visible nodules $(>1 \mathrm{~mm})$ of various colors observed at autopsy, were histologically classified based on Mouse tumor classification criteria [17] as eosinophilic or clear cells nodules, adenomas, cholangiomas, cholangiocellular carcinoma or hepatocellular carcinoma (HCC).

\subsection{Immunohistochemistry}

To evaluate the expression of selected markers in liver samples submitted to the microarray experiment, immunolabeling for glutamine synthetase, $\beta$-catenin, and TGF- $\beta 1$ was performed. Liver tissue sections were deparaffinized and subjected to degrading alcohol gradient and treated for antigen retrieval before staining. Afterwards, $3 \%$ hydrogen peroxide $\left(\mathrm{H}_{2} \mathrm{O}_{2}\right)$ was used for endogenous peroxidase quenching, non-specific staining was blocked using 5\% normal goat serum (Sigma-Aldrich, St. Louis, MO, USA) for $1 \mathrm{~h}$ at room temperature. Incubation with primary, anti-glutamine synthetase (BD Biosciences, San Jose, CA, USA; 1:1000 dilution), anti- $\beta$-catenin (BD Biosciences, San Jose, CA, USA; 1:1000 dilution) and anti-TGF- $\beta 1$ (Promega, Madison, WI, USA; 1:500 dilution) antibodies in $1 \%$ goat serum in $0.1 \%$ Tris buffered saline with Tween 20 (TBST) was performed overnight at $4{ }^{\circ} \mathrm{C}$. We used the DAKO EnVision Detection System (Agilent Technologies DAKO, Glostrup, Denmark) for antibody detection. The sections were subsequently counterstained with hematoxylin.

\subsection{Analysis of Plasma Parameters}

Total and HDL cholesterol, free fatty acids (FFA), triglycerides (TG), alanine aminotransferase (ALT), and aspartate aminotransferase (AST) were analyzed with the Architect ci8200 analyzer (Abbott Diagnostics, Abbott Park, IL, USA). The concentration of lipid parameters were measured as $\mathrm{mmol} / \mathrm{L}$ and a linear regression model Age+Genotype+Sex+Age:Sex was used to fit the concentrations of individual lipids. The activity of ALT and AST were given in $\mu \mathrm{kat} / \mathrm{L}$ and a linear regression model Age+Genotype+Sex+Genotype:Sex was used to fit their activity individually. ANOVA type III test was used to estimate the statistical significance of the effects and the Holm method was used to control the family-wise error rate (FWER) at $\alpha=0.05$.

\subsection{Liver Sterol Analysis}

Sterols isolation: Sterol intermediates were isolated with the protocol described previously [76]. Folch solution with samples corresponding to $80 \mathrm{mg}$ of liver tissue was transfer to fresh tubes and $200 \mathrm{ng}$ of internal standard Lathosterol-D7 (Avanti Polar Lipids, Alabaster, AL, USA) was added. Final samples were dissolved in $250 \mu \mathrm{L}$ of methanol and transferred to HPLC vials for analysis. LC-MS analysis was performed as described [77]. Briefly, sterols were separated on combined columns of Luna ${ }^{\circledR}$ $3 \mu \mathrm{m}$ PFP (2) $100 \AA$ (100 mm and $150 \mathrm{~mm}$ length) on a Shimadzu Nexera XR HPLC. For mobile phase, $80 \%$ methanol, $10 \%$ water, $10 \%$ 1-propanol, and $0.05 \%$ Formic acid were used in the isocratic condition. HPLC was coupled with the Sciex Triple Quad 3500 mass spectrometer for detection. For ionization, APCI (Atmospheric-pressure chemical ionization) was used in positive mode and detection was made in MRM (Multiple reaction monitoring) mode. The sample concentration was normalized on internal standard (Lathosterol-d7) and sterol concentrations calculated to corresponding standards from Avanti Lipids. We measured the following sterol intermediates, with MRM in brackets: Zymosterol* (367/215), 24-dehydrolathosterol* (367/215), 7-dehydrodesmosterol* (365/199), Desmosterol (367/215), Zymostenol (369/215), Lathosterol-d7 (376/215), FF-MAS* (393/214), T-MAS* (395/243), 
Cholesterol (369/215), Lanosterol (409/191) and 24,25-dihydrolanosterol (411/191). *Sterol concentration was too low to quantify.

\subsection{Gene Expression Profiling and Network Analysis}

Transcriptome analysis of liver samples of Cyp51 WT and KO mice of both sexes was performed using Affymetrix GeneChip ${ }^{\mathrm{TM}}$ Mouse Gene 2.0 ST arrays and validated by qPCR (primers listed in Table S14; further details in Appendix A. The same mice and groups were used for plasma analysis (Table A2). For qPCR, a linear regression model Age+Genotype+Sex+Age:Genotype was used to fit normalized expression data of cholesterol-related genes Hmgcr, Sqle, Lss, Cyp51, Nsdhl and Dhcr7, and a reduced additive model Age+Genotype+Sex was used to fit data of fatty acid-related genes Cyp7a1, Cyp8b1 and Cyp27a1. ANOVA type III test was used to estimate the statistical significance of the effects and Holm method was used to control FWER at $\alpha=0.05$.

A total of 20 Affymetrix GeneChip ${ }^{\mathrm{TM}}$ Mouse Gene 2.0 ST Arrays (Affymetrix, Santa Clara, CA, USA) with liver samples were processed. From each of the Cyp51 KO livers, two samples were taken: tumor and surrounding tissue. Altogether, 6 tumor and 6 corresponding surrounding-tissue samples were obtained from Cyp51 KO mice (Table A3). The data were analysed using R/limma, controlling the false discovery rate at $\alpha=0.05$, and deposited to Gene Expression Omnibus (GEO) under accession number GSE127772. KEGG PATHWAY and TRANSFAC databases were used for functional enrichment studies. Gene expression data from 19 weeks (W) [16] WT and KO mice of both sexes were used to determine processes that contribute to tumor progression from 19W towards 24M. Network diagrams were created using NetworkAnalyst and STRING. The proposed mechanistic scheme was designed from data obtained from mice experiments and aligned to human literature data to increase their relevance.

\section{Conclusions}

The hallmark of cancer cells is metabolic reprogramming, causing new cellular demands in selective survival and growth. Metabolic reprogramming of hepatic cholesterol synthesis leads to female prevalent hepatocellular carcinoma during aging. Inactivation of Cyp51 affected multiple signalling pathways and transcription factors in a sex-dependent manner, resulting in metabolic-related HCC. The female-specific cholesterol-related mechanism of hepatocarcinogenesis is very similar to the human data and is in line with the fact that the liver is a sex-dimorphic metabolic organ and a major organ of cholesterol homeostasis. Since hepatocellular carcinoma is increasing in post-menopausal women, the female-specific aspects of liver cancer progression, diagnosis and treatment should become a central focus of research.

Supplementary Materials: The following are available online at http://www.mdpi.com/2072-6694/12/11/3302/s1, in files (1) Supplementary Figures and (2) Supplementary Tables. Figure S1: Patho-histological features of Cyp51 KO mice. Figure S2: The KO mice liver and body weights at different ages, both sexes. Figure S3: Immunohistochemical expression of TGF- $\beta 1$ and $\beta$-catenin. Figure S4: qPCR expression profiles of TGF- $\beta$ and Wnt signalling markers. Figure S5: TF networks of $24 \mathrm{M} \mathrm{KO}$ mice. Table S1: List of gene sequences for qPCR validation. Table S2: Female Cyp51 KO mice had a higher incidence of liver tumors as males. Table S3: Differences between mouse liver/body/relative weights. Table S4: A heat-map of cholesterol and bile acid synthesis DNA-microarray gene expression in male and female KO mice. Table S5: The long-term ablation of Cyp51 in hepatocytes influence at gene and metabolic levels. Table S6: DNA-microarray gene expression tables. Table S7: KEGG pathways expression tables deduced from DNA-microarray gene expression pattern. Table S8: Transcription factors expression tables deduced from DNA-microarray gene expression pattern. Table S9: A clear sex imbalance in gene expression in 24M KOs. Table S10: List of sex-specific top 10 statistically significant down- and up-regulated genes (deduced from Table S6) in Cyp51 KO mice, aged 19 weeks and 24 months. Table S11: List of sex-specific top 10 statistically significant down- and up-regulared TFs (deduced from Table S8) in Cyp51 KO mice, aged 19 weeks and 24 months. Table S12: Target genes of TFs RORC and SOX9. Table S13: COSMIC database information of CYP51A1 point mutations in humans. Table S14: The positive data (mutated samples) for selected gene CYP51A1 from COSMIC database. The datasets generated during the current study are available in the Gene Expression Omnibus database (https://www.ncbi.nlm.nih.gov/geo/) under accession number GSE127772 and GSE58271. 
Author Contributions: Conceptualization, D.R.; methodology, K.B.C., G.L., M.P., J.J., Ž.U. and C.S.; software, P.J.; validation, K.B.C. and C.S.; formal analysis, P.J.; investigation, K.B.C.; resources, D.R., and M.M.-S.; data curation, P.J.; writing—original draft preparation, K.B.C., M.P., J.J., T.R., and D.R.; writing—review and editing, all authors; visualization, K.B.C.; supervision, D.R.; project administration, D.R.; funding acquisition, D.R. All authors have read and agreed to the published version of the manuscript.

Funding: This research was funded by the Slovenian Research Agency (ARRS) program grants P1-0104, P1-0390, and projects J1-9176 and BI-DE/17-19-8. Kaja Blagotinšek Cokan, Gregor Lorbek, and Žiga Urlep were supported by the graduate fellowship of ARRS.

Acknowledgments: We thank Ksenija Kodra, Helena Klavžar, Marjan Kužnik, and Doris Mahn for their help with experiments. We acknowledge Simon Horvat and Rok Keber for their previous contribution to the development of the Cyp51 knock-out mouse model. Thanks also to Nejc Nadižar for help in statistical data evaluation and figure preparation and Petra Ivanuša for help with sterol isolation. Thanks to collaborators Irena Vovk and Mitja Križman for LC-MS method development.

Conflicts of Interest: The authors declare no conflict of interest.

\section{Abbreviations}

\begin{tabular}{ll} 
CYP51 & Lanosterol 14 $\alpha$-demethylase \\
MAFLD & Metabolic Associated Fatty Liver Disease \\
NAFLD & Non-Alcoholic Fatty Liver Disease \\
HCC & Hepatocellular Carcinoma \\
WT & Wild-Type \\
KO & Knock-Out \\
M & Month \\
W & Week \\
H\&E & Hematoxylin and Eosin \\
SR & Sirius Red \\
FFA & Fatty Free Acids \\
TG & Triglycerides \\
BA & Bile Acids \\
CHOL & Cholesterol \\
HDL & High-Density Lipoprotein \\
ALT & Alanine Transaminase \\
AST & Aspartate Transaminase \\
LC-MS & Liquid Chromatography-Mass Spectrometry \\
HMGCR & 3-hydroxy-3-methyl-glutaryl-coenzyme A reductase \\
DHCR24 & 24-dehydrocholesterol reductase \\
GEO & Gene Expression Omnibus \\
DE & Differentially Expression \\
ECM & Extracellular Matrix \\
KEGG & Kyoto Encyclopedia of Genes and Genomes \\
EMT & Epithelial to Mesenchymal Transition \\
TF & Transcription Factor \\
& \\
\hline &
\end{tabular}




\section{Appendix A}

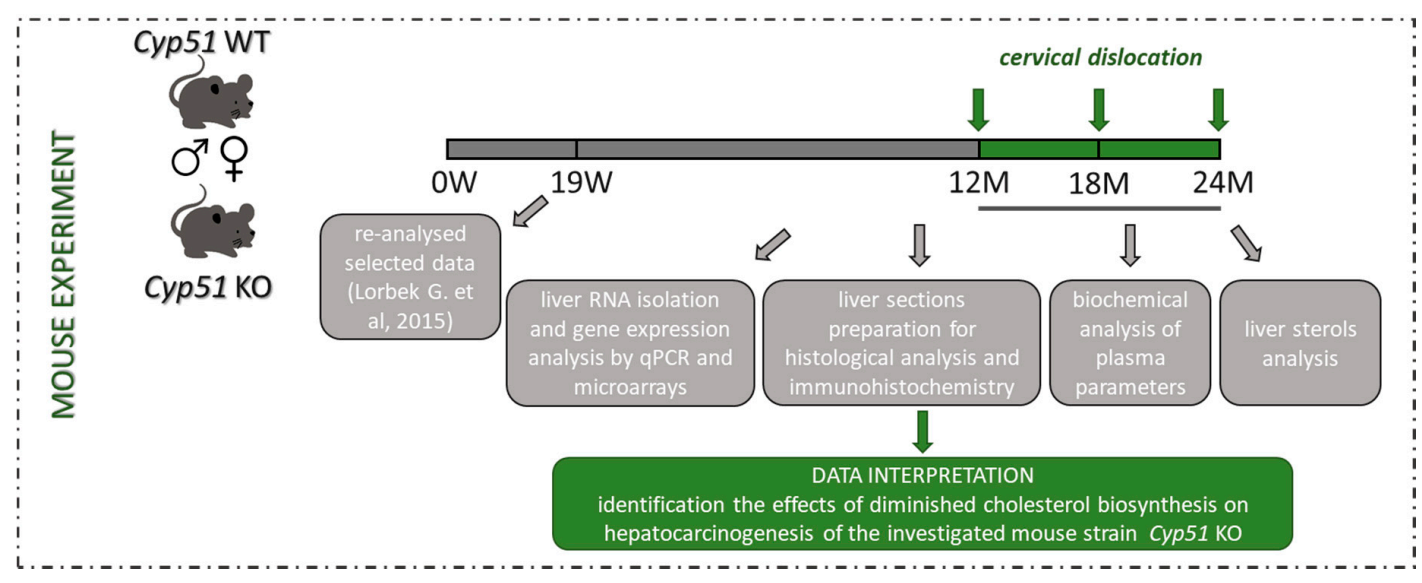

Figure A1. Experimental design. Experiments were performed in female and male Cyp51 liver conditional knock-out mice at age of 12,18, and 24 months, and the wild-type controls. Collected data from livers and blood samples were aligned to data of the 19 weeks old mice.

Table A1. List of negatively tested microorganisms in mice in routine health microbiological monitoring.

\begin{tabular}{cc}
\hline \multicolumn{1}{c}{ List of Negatively Tested Microorganisms } \\
\hline Citrobacter rodentium & Minute virus of mice \\
\hline Corynebacterium kutscheri & Mouse hepatitis virus \\
\hline Klebsiella oxytoca & Mouse parvovirus (rVP2) \\
\hline Klebsiella pneumoniae & Mouse rotavirus/EDIM \\
\hline Pseudomonas aeruginosa & Mycoplasma pulmonis \\
\hline Salmonela spp. & Pneumonia virus of mice \\
\hline Staphylococcus aureus & Reovirus type 3 \\
\hline Streptobacillus moniformis & Sendai virus \\
\hline Streptococci beta-haemolytic Group A & Theiler's encephalomyelitis virus (GD VII) \\
\hline Streptococci beta-haemolytic Group B & Myobia musculi/Radfordia sp. \\
\hline Streptococci beta-haemolytic Group C & Other ectoparasites \\
\hline Streptococci beta-haemolytic Group $G$ & Aspiculuris tetraptera \\
\hline Streptococcus pneumoniae & Cryptosporidium spp. \\
\hline Adenovirus FL & Entamoeba spp. \\
\hline Adenovirus K87 & Giardia spp. \\
\hline Clostridium piliforme & Helicobacter bilis \\
\hline Ectromelia virus & Spironucleus spp. \\
\hline General parvovirus ( $r$ NS-1) & Syphacia obvelata \\
\hline Lymocytic choriomeningitisvirus & \\
\hline
\end{tabular}

\section{Plasma Analysis}

Mice were placed into groups according to their age (12, 18 and 24 months, 24 mice per group), genotype (37 Cyp51 WT, 35 Cyp51 KO), and sex (40 female, 32 male). 72 mouse samples (4-7 samples per group) were analysed (Table A2). 
Table A2. Number of mouse samples for plasma and RT-qPCR gene expression analysis.

\begin{tabular}{ccccc}
\hline \multicolumn{2}{c}{ Mouse Samples } & \multicolumn{3}{c}{ Age } \\
\hline Genotype & Sex & $\mathbf{1 2 M}$ & $\mathbf{1 8 M}$ & $\mathbf{2 4 M}$ \\
\hline \multirow{2}{*}{ Cyp51 WT } & female & 7 & 7 & 7 \\
\multirow{2}{*}{ Cyp51 KO } & male & 5 & 5 & 6 \\
& female & 5 & 7 & 7 \\
& male & 7 & 5 & 4 \\
\hline
\end{tabular}

\section{Gene Expression Analysis}

The same mice and groups were used as for plasma analysis (Table A2). Total RNA was isolated from $30 \mu \mathrm{g}$ of the liver using TRI Reagent (Sigma-Aldrich, St. Louis, MO, USA) procedure. RNA concentration and purity of each sample were assayed using NanoDrop 1000 Spectrophotometer (Thermo Fischer Scientific, Waltham, MA, USA). The RNA quality was checked with Agilent 2100 BioAnalyzer (Agilent Technologies, Santa Clara, CA, USA). Before cDNA synthesis, all liver RNA samples $(2 \mu \mathrm{g})$ were treated with amplification grade DNase I (Roche, Basel, Switzerland), and reverse-transcribed with the Transcriptor Universal cDNA Master (Roche, Basel, Switzerland) according to manufacturer's instructions.

Real-time quantitative reverse transcription polymerase chain reaction (RT-qPCR) was performed using a Roche LightCycler 480 (Roche, Basel, Switzerland). In all experiments, the PCR reaction consisted of $2.5 \mu \mathrm{L}$ SYBR Green I Master (Roche, Basel, Switzerland), $0.75 \mu \mathrm{L}$ of cDNA template, $0.6 \mu \mathrm{L}$ of $2.5 \mu \mathrm{M}$ random primer mix and $1.15 \mu \mathrm{L}$ of RNAse-free water in a final volume of $5 \mu \mathrm{L}$. All experiments were carried out in three technical replicates for each of the sample using 384-well plates. PCR was performed with the following parameters: $10 \mathrm{~min}$ incubation at $95^{\circ} \mathrm{C}$ followed by 45 cycles of $10 \mathrm{~s}$ at $95^{\circ} \mathrm{C}, 20 \mathrm{~s}$ at $58{ }^{\circ} \mathrm{C}$ and $20 \mathrm{~s}$ at $72{ }^{\circ} \mathrm{C}$. Utp 6 and Hmbs were chosen as internal reference genes in mice liver for normalization of gene expression data using NormFinder [78]. The relative expression ratio was calculated using the ddCp as previously described [79]. The list of primer sequences used in RT-qPCR is provided under Table S1: oligonucleotide sequences.

\section{Microarray-Based Gene Expression Profiling}

Table A3. Number of $24 \mathrm{M}$ mouse samples for DNA-microarray analysis. $\leftrightarrow$ indicates related samples, i.e., different tissue samples obtained from individual mice.

\begin{tabular}{cccccc}
\hline & \multicolumn{4}{c}{ Genotype } \\
\cline { 3 - 6 } 24M Mouse Samples & Cyp51 & \multicolumn{3}{c}{ Cyp51 KO } \\
& WT & \multicolumn{3}{c}{ Tissue } \\
& Normal & \multicolumn{2}{c}{ Surrounding } & HCC \\
\hline \multirow{2}{*}{ Sex } & Female & 4 & 4 & $\leftrightarrow$ & 4 \\
& Male & 4 & 2 & $\leftrightarrow$ & 2 \\
\hline
\end{tabular}

The experiment was performed with $500 \mathrm{ng}$ starting concentration of total RNA with a purity (absorbance ratio A260/280) $>1.8$ and quality $(\mathrm{RIN})>4.5$. The procedure was done as described previously $[10,16]$ and considering the manufacturer's instructions. Briefly, after overnight hybridization at $45^{\circ} \mathrm{C}$ and $60 \mathrm{rpm} / \mathrm{min}$ microarrays were washed and stained using GeneChip Fluidics Station 450 and scanned on Affymetrix GeneChip Scanner 3000 7G. Image analysis and first quality check were done using the Affymetrix Expression Console ${ }^{\mathrm{TM}}$ version 1.3.

Further quality control and analysis were performed using $\mathrm{R}$ and Bioconductor software packages. RMA algorithm from package oligo [80] was used for the normalization of raw expression data. Package arrayQualityMetrics [81] was used to check additional quality control and assessment of potential outliers before and after normalization. Raw (CEL) as well as normalized data were deposited to GEO under accession number GSE127772 (https://www.ncbi.nlm.nih.gov/geo/query/acc.cgi?acc=GSE127772). Package limma [82] was used to fit individual normalized gene expression and gene set enrichment data using a linear regression model Genotype+Sex+Genotype:Sex. Empirical Bayes statistics was used to estimate the significance of the effects for each gene (gene set) and Benjamini-Hochberg procedure was used to control false discovery rate (FDR) at $\alpha=0.05$ accounting for the number of tested genes (gene sets) and to infer differential expression of genes (enrichment of gene sets).

KEGG PATHWAY [82] and TRANSFAC database were used for functional enrichment studies [83]. Gene sets containing over 5 elements were constructed and tested for enrichment using the PGSEA package [84]. In the case of transcription factor enrichment, factors were merged based on their ID irrespective of their binding sites.

Gene expression data from 19 weeks $(19 \mathrm{~W})$ old hepatocyte-specific Cyp51 KO mice (Cyp51 flox/flox; Alb-Cre or LKO; i.e., KO) and their WT littermates (Cyp51 floxflox or LWT; i.e., WT) following standard rodent diet (low-fat 
no-cholesterol, LFnC) was analysed to determine processes that contribute to tumor progression from $19 \mathrm{~W}$ towards 24M. Data is available from GEO under accession number GSE58271 (https:/www.ncbi.nlm.nih.gov/geo/query/acc. cgi) [16]. Data was preprocessed, annotation updated and analyzed using the same procedure as described above. A linear regression model Genotype+Sex+Genotype:Sex was used to fit individual normalized gene expression and gene set enrichment data from mouse samples shown in Table A4.

Table A4. Number of 19W mouse samples from GSE58271 that were included to determine processes that contribute to tumor progression from $19 \mathrm{~W}$ towards $24 \mathrm{M}$.

\begin{tabular}{lccc}
\hline \multirow{2}{*}{ 19W Mouse Samples } & \multicolumn{2}{c}{ Genotype } \\
\cline { 3 - 4 } & & WT & KO \\
\hline \multirow{2}{*}{ Sex } & Female & 3 & 3 \\
& Male & 3 & 3 \\
\hline
\end{tabular}

Network diagrams of differences in gene expression in females and males (Figure 4C) were created using the NetworkAnalyst program [19]. For the generation and visualization of transcription factor networks (Figure S5), the list of female and male DEG was mapped to the "database" libraries of the STRING online software [27] using medium confidence score (0.4).

\section{References}

1. Eslam, M.; Sanyal, A.J.; George, J.; International Consensus Panel. MAFLD: A consensus-driven proposed nomenclature for metabolic associated fatty liver disease. Gastroenterology 2020. [CrossRef]

2. Nakagawa, H.; Hayata, Y.; Kawamura, S.; Yamada, T.; Fujiwara, N.; Koike, K. Lipid Metabolic Reprogramming in Hepatocellular Carcinoma. Cancers 2018, 10, 447. [CrossRef]

3. Burra, P.; De Martin, E.; Gitto, S.; Villa, E. Influence of age and gender before and after liver transplantation. Liver Transplant. Off. Publ. Am. Assoc. Study Liver Dis. Int. Liver Transplant. Soc. 2013, 19, 122-134. [CrossRef]

4. Chua, N.K.; Coates, H.W.; Brown, A.J. Cholesterol, cancer, and rebooting a treatment for athlete's foot. Sci. Transl. Med. 2018, 10. [CrossRef]

5. Horvat, S.; McWhir, J.; Rozman, D. Defects in cholesterol synthesis genes in mouse and in humans: Lessons for drug development and safer treatments. Drug Metab. Rev. 2011, 43, 69-90. [CrossRef]

6. Huang, B.; Song, B.L.; Xu, C. Cholesterol metabolism in cancer: Mechanisms and therapeutic opportunities. Nat. Metab. 2020, 2, 132-141. [CrossRef] [PubMed]

7. Che, L.; Chi, W.; Qiao, Y.; Zhang, J.; Song, X.; Liu, Y.; Li, L.; Jia, J.; Pilo, M.G.; Wang, J.; et al. Cholesterol biosynthesis supports the growth of hepatocarcinoma lesions depleted of fatty acid synthase in mice and humans. Gut 2020, 69, 177-186. [CrossRef]

8. Zhu, W.W.; Lu, M.; Wang, X.Y.; Zhou, X.; Gao, C.; Qin, L.X. The fuel and engine: The roles of reprogrammed metabolism in metastasis of primary liver cancer. Genes Dis. 2020, 7, 299-307. [CrossRef]

9. Liu, D.; Wong, C.C.; Fu, L.; Chen, H.; Zhao, L.; Li, C.; Zhou, Y.; Zhang, Y.; Xu, W.; Yang, Y.; et al. Squalene epoxidase drives NAFLD-induced hepatocellular carcinoma and is a pharmaceutical target. Sci. Transl. Med. 2018, 10. [CrossRef]

10. Urlep, Z.; Lorbek, G.; Perse, M.; Jeruc, J.; Juvan, P.; Matz-Soja, M.; Gebhardt, R.; Bjorkhem, I.; Hall, J.A.; Bonneau, R.; et al. Disrupting Hepatocyte Cyp51 from Cholesterol Synthesis Leads to Progressive Liver Injury in the Developing Mouse and Decreases RORC Signalling. Sci. Rep. 2017, 7, 40775. [CrossRef] [PubMed]

11. Lorbek, G.; Urlep, Z.; Rozman, D. Pharmacogenomic and personalized approaches to tackle nonalcoholic fatty liver disease. Pharmacogenomics 2016, 17, 1273-1288. [CrossRef]

12. Wilson, M.A.; Buetow, K.H. Novel Mechanisms of Cancer Emerge When Accounting for Sex as a Biological Variable. Cancer Res. 2020, 80, 27-29. [CrossRef]

13. Li, C.H.; Haider, S.; Shiah, Y.J.; Thai, K.; Boutros, P.C. Sex Differences in Cancer Driver Genes and Biomarkers. Cancer Res. 2018, 78, 5527-5537. [CrossRef] [PubMed]

14. Lonardo, A.; Nascimbeni, F.; Ballestri, S.; Fairweather, D.; Win, S.; Than, T.A.; Abdelmalek, M.F.; Suzuki, A. Sex Differences in Nonalcoholic Fatty Liver Disease: State of the Art and Identification of Research Gaps. Hepatology 2019, 70, 1457-1469. [CrossRef] 
15. Cvitanovic Tomas, T.; Urlep, Z.; Moskon, M.; Mraz, M.; Rozman, D. LiverSex Computational Model: Sexual Aspects in Hepatic Metabolism and Abnormalities. Front. Physiol. 2018, 9, 360. [CrossRef]

16. Lorbek, G.; Perse, M.; Jeruc, J.; Juvan, P.; Gutierrez-Mariscal, F.M.; Lewinska, M.; Gebhardt, R.; Keber, R.; Horvat, S.; Bjorkhem, I.; et al. Lessons from hepatocyte-specific Cyp51 knockout mice: Impaired cholesterol synthesis leads to oval cell-driven liver injury. Sci. Rep. 2015, 5, 8777. [CrossRef]

17. Deschl, U.; Cattley, R.C.; Harada, T.; Kuttler, K.; Hailey, J.R.; Harting, F.; Leblanc, B.; Marsman, D.S.; Shirai, T. Liver, Gallbladder, and Exocrine Pancreas. In International Classification of Rodent Tumors: The Mouse; Mohr, U., Ed.; Springer: Berlin/Heidelberg Germany; New York, NY, USA, 2001; pp. 59-86.

18. Salleng, K.J.; Revetta, F.L.; Deane, N.G.; Washington, M.K. The Applicability of a Human Immunohistochemical Panel to Mouse Models of Hepatocellular Neoplasia. Comp. Med. 2015, 65, 398-408.

19. Xia, J.; Benner, M.J.; Hancock, R.E. NetworkAnalyst-integrative approaches for protein-protein interaction network analysis and visual exploration. Nucleic Acids Res. 2014, 42, W167-W174. [CrossRef]

20. Leung, C.O.; Mak, W.N.; Kai, A.K.; Chan, K.S.; Lee, T.K.; Ng, I.O.; Lo, R.C. Sox9 confers stemness properties in hepatocellular carcinoma through Frizzled-7 mediated Wnt/beta-catenin signaling. Oncotarget 2016, 7, 29371-29386. [CrossRef]

21. Steensels, S.; Qiao, J.; Ersoy, B.A. Transcriptional Regulation in Non-Alcoholic Fatty Liver Disease. Metabolites 2020, 10, 283. [CrossRef]

22. Mello, T.; Materozzi, M.; Galli, A. PPARs and Mitochondrial Metabolism: From NAFLD to HCC. PPAR Res. 2016, 2016, 7403230. [CrossRef]

23. Kurokawa, T.; Shimomura, Y.; Bajotto, G.; Kotake, K.; Arikawa, T.; Ito, N.; Yasuda, A.; Nagata, H.; Nonami, T.; Masuko, K. Peroxisome proliferator-activated receptor alpha (PPARalpha) mRNA expression in human hepatocellular carcinoma tissue and non-cancerous liver tissue. World J. Surg. Oncol. 2011, 9, 167. [CrossRef]

24. Wang, S.; Kang, X.; Cao, S.; Cheng, H.; Wang, D.; Geng, J. Calcineurin/NFATc1 pathway contributes to cell proliferation in hepatocellular carcinoma. Dig. Dis. Sci. 2012, 57, 3184-3188. [CrossRef]

25. Li, L.; Yu, J.; Duan, Z.; Dang, H.X. The effect of NFATc1 on vascular generation and the possible underlying mechanism in epithelial ovarian carcinoma. Int. J. Oncol. 2016, 48, 1457-1466. [CrossRef]

26. Santori, F.R.; Huang, P.; van de Pavert, S.A.; Douglass, E.F., Jr.; Leaver, D.J.; Haubrich, B.A.; Keber, R.; Lorbek, G.; Konijn, T.; Rosales, B.N.; et al. Identification of natural RORgamma ligands that regulate the development of lymphoid cells. Cell Metab. 2015, 21, 286-298. [CrossRef]

27. Szklarczyk, D.; Morris, J.H.; Cook, H.; Kuhn, M.; Wyder, S.; Simonovic, M.; Santos, A.; Doncheva, N.T.; Roth, A.; Bork, P.; et al. The STRING database in 2017: Quality-controlled protein-protein association networks, made broadly accessible. Nucleic Acids Res. 2017, 45, D362-D368. [CrossRef]

28. Smeuninx, B.; Boslem, E.; Febbraio, M.A. Current and Future Treatments in the Fight Against Non-Alcoholic Fatty Liver Disease. Cancers 2020, 12, 1714. [CrossRef]

29. Xiao, Y.B.; Cai, S.H.; Liu, L.L.; Yang, X.; Yun, J.P. Decreased expression of peroxisome proliferator-activated receptor alpha indicates unfavorable outcomes in hepatocellular carcinoma. Cancer Manag. Res. 2018, 10, 1781-1789. [CrossRef] [PubMed]

30. Kovac, U.; Skubic, C.; Bohinc, L.; Rozman, D.; Rezen, T. Oxysterols and Gastrointestinal Cancers Around the Clock. Front. Endocrinol. 2019, 10, 483. [CrossRef]

31. Moren, A.; Bellomo, C.; Tsubakihara, Y.; Kardassis, D.; Mikulits, W.; Heldin, C.H.; Moustakas, A. LXRalpha limits TGFbeta-dependent hepatocellular carcinoma associated fibroblast differentiation. Oncogenesis 2019, 8, 36. [CrossRef] [PubMed]

32. Yang, J.D.; Nakamura, I.; Roberts, L.R. The tumor microenvironment in hepatocellular carcinoma: Current status and therapeutic targets. Semin. Cancer Biol. 2011, 21, 35-43. [CrossRef]

33. Walker, C.; Mojares, E.; Del Rio Hernandez, A. Role of Extracellular Matrix in Development and Cancer Progression. Int. J. Mol. Sci. 2018, 19, 3028. [CrossRef]

34. Jiang, N.; Dai, Q.; Su, X.; Fu, J.; Feng, X.; Peng, J. Role of PI3K/AKT pathway in cancer: The framework of malignant behavior. Mol. Biol. Rep. 2020, 47, 4587-4629. [CrossRef] [PubMed]

35. Niavarani, S.R.; Lawson, C.; Bakos, O.; Boudaud, M.; Batenchuk, C.; Rouleau, S.; Tai, L.H. Lipid accumulation impairs natural killer cell cytotoxicity and tumor control in the postoperative period. BMC Cancer 2019, 19, 823. [CrossRef]

36. Goldberg, I.J.; Eckel, R.H.; Abumrad, N.A. Regulation of fatty acid uptake into tissues: Lipoprotein lipaseand CD36-mediated pathways. J. Lipid Res. 2009, 50, S86-S90. [CrossRef] 
37. Nath, A.; Li, I.; Roberts, L.R.; Chan, C. Elevated free fatty acid uptake via CD36 promotes epithelial-mesenchymal transition in hepatocellular carcinoma. Sci. Rep. 2015, 5, 14752. [CrossRef]

38. Takeda, Y.; Kang, H.S.; Freudenberg, J.; DeGraff, L.M.; Jothi, R.; Jetten, A.M. Retinoic acid-related orphan receptor gamma (RORgamma): A novel participant in the diurnal regulation of hepatic gluconeogenesis and insulin sensitivity. PLoS Genet. 2014, 10, e1004331. [CrossRef]

39. Massa, M.L.; Gagliardino, J.J.; Francini, F. Liver glucokinase: An overview on the regulatory mechanisms of its activity. IUBMB Life 2011, 63, 1-6. [CrossRef] [PubMed]

40. Athwal, V.S.; Pritchett, J.; Llewellyn, J.; Martin, K.; Camacho, E.; Raza, S.M.; Phythian-Adams, A.; Birchall, L.J.; Mullan, A.F.; $\mathrm{Su}, \mathrm{K}$.; et al. SOX9 predicts progression toward cirrhosis in patients while its loss protects against liver fibrosis. EMBO Mol. Med. 2017, 9, 1696-1710. [CrossRef]

41. Athwal, V.S.; Pritchett, J.; Martin, K.; Llewellyn, J.; Scott, J.; Harvey, E.; Zaitoun, A.M.; Mullan, A.F.; Zeef, L.A.H.; Friedman, S.L.; et al. SOX9 regulated matrix proteins are increased in patients serum and correlate with severity of liver fibrosis. Sci. Rep. 2018, 8, 17905. [CrossRef]

42. Infantino, V.; Dituri, F.; Convertini, P.; Santarsiero, A.; Palmieri, F.; Todisco, S.; Mancarella, S.; Giannelli, G.; Iacobazzi, V. Epigenetic upregulation and functional role of the mitochondrial aspartate/glutamate carrier isoform 1 in hepatocellular carcinoma. Biochim. Biophys. Acta Mol. Basis Dis. 2019, 1865, 38-47. [CrossRef]

43. Cruz, P.M.; Mo, H.; McConathy, W.J.; Sabnis, N.; Lacko, A.G. The role of cholesterol metabolism and cholesterol transport in carcinogenesis: A review of scientific findings, relevant to future cancer therapeutics. Front. Pharmacol. 2013, 4, 119. [CrossRef]

44. Acimovic, J.; Rozman, D. Steroidal triterpenes of cholesterol synthesis. Molecules 2013, 18, $4002-4017$. [CrossRef]

45. Guy, J.; Peters, M.G. Liver disease in women: The influence of gender on epidemiology, natural history, and patient outcomes. Gastroenterol. Hepatol. (N. Y.) 2013, 9, 633-639.

46. Kohi, M.P. Gender-Related Differences in Hepatocellular Carcinoma: Does Sex Matter? J. Vasc. Interv. Radiol. JVIR 2016, 27, 1338-1341. [CrossRef]

47. Hartwell, H.J.; Petrosky, K.Y.; Fox, J.G.; Horseman, N.D.; Rogers, A.B. Prolactin prevents hepatocellular carcinoma by restricting innate immune activation of c-Myc in mice. Proc. Natl. Acad. Sci. USA 2014, 111, 11455-11460. [CrossRef]

48. Keber, R.; Motaln, H.; Wagner, K.D.; Debeljak, N.; Rassoulzadegan, M.; Acimovic, J.; Rozman, D.; Horvat, S. Mouse knockout of the cholesterogenic cytochrome P450 lanosterol 14alpha-demethylase (Cyp51) resembles Antley-Bixler syndrome. J. Biol. Chem. 2011, 286, 29086-29097. [CrossRef] [PubMed]

49. Gillespie, R.L.; Urquhart, J.; Anderson, B.; Williams, S.; Waller, S.; Ashworth, J.; Biswas, S.; Jones, S.; Stewart, F.; Lloyd, I.C.; et al. Next-generation Sequencing in the Diagnosis of Metabolic Disease Marked by Pediatric Cataract. Ophthalmology 2016, 123, 217-220. [CrossRef]

50. Patel, N.; Anand, D.; Monies, D.; Maddirevula, S.; Khan, A.O.; Algoufi, T.; Alowain, M.; Faqeih, E.; Alshammari, M.; Qudair, A.; et al. Novel phenotypes and loci identified through clinical genomics approaches to pediatric cataract. Hum. Genet. 2017, 136, 205-225. [CrossRef] [PubMed]

51. Rezen, T.; Ogris, I.; Sever, M.; Merzel, F.; Golic Grdadolnik, S.; Rozman, D. Evaluation of Selected CYP51A1 Polymorphisms in View of Interactions with Substrate and Redox Partner. Front. Pharmacol. 2017, 8, 417. [CrossRef]

52. Lewinska, M.; Zelenko, U.; Merzel, F.; Golic Grdadolnik, S.; Murray, J.C.; Rozman, D. Polymorphisms of CYP51A1 from cholesterol synthesis: Associations with birth weight and maternal lipid levels and impact on CYP51 protein structure. PLoS ONE 2013, 8, e82554. [CrossRef]

53. Michailidou, K.; Lindstrom, S.; Dennis, J.; Beesley, J.; Hui, S.; Kar, S.; Lemacon, A.; Soucy, P.; Glubb, D.; Rostamianfar, A.; et al. Association analysis identifies 65 new breast cancer risk loci. Nature 2017, 551, 92-94. [CrossRef]

54. Yashin, A.I.; Wu, D.; Arbeeva, L.S.; Arbeev, K.G.; Kulminski, A.M.; Akushevich, I.; Kovtun, M.; Culminskaya, I.; Stallard, E.; Li, M.; et al. Genetics of aging, health, and survival: Dynamic regulation of human longevity related traits. Front. Genet. 2015, 6, 122. [CrossRef]

55. Haines, T.H. Do sterols reduce proton and sodium leaks through lipid bilayers? Prog. Lipid Res. 2001, 40, 299-324. [CrossRef]

56. Jiang, J.T.; Xu, N.; Zhang, X.Y.; Wu, C.P. Lipids changes in liver cancer. J. Zhejiang Univ. Sci. B 2007, 8, 398-409. [CrossRef] 
57. Hubler, Z.; Allimuthu, D.; Bederman, I.; Elitt, M.S.; Madhavan, M.; Allan, K.C.; Shick, H.E.; Garrison, E.; Molly, T.K.; Factor, D.C.; et al. Accumulation of 8,9-unsaturated sterols drives oligodendrocyte formation and remyelination. Nature 2018, 560, 372-376. [CrossRef]

58. Gabitova, L.; Restifo, D.; Gorin, A.; Manocha, K.; Handorf, E.; Yang, D.H.; Cai, K.Q.; Klein-Szanto, A.J.; Cunningham, D.; Kratz, L.E.; et al. Endogenous Sterol Metabolites Regulate Growth of EGFR/KRAS-Dependent Tumors via LXR. Cell Rep. 2015, 12, 1927-1938. [CrossRef] [PubMed]

59. Phillips, R.E.; Yang, Y.; Smith, R.C.; Thompson, B.M.; Yamasaki, T.; Soto-Feliciano, Y.M.; Funato, K.; Liang, Y.; Garcia-Bermudez, J.; Wang, X.; et al. Target identification reveals lanosterol synthase as a vulnerability in glioma. Proc. Natl. Acad. Sci. USA 2019. [CrossRef]

60. Chen, L.; Ma, M.Y.; Sun, M.; Jiang, L.Y.; Zhao, X.T.; Fang, X.X.; Man Lam, S.; Shui, G.H.; Luo, J.; Shi, X.J.; et al. Endogenous sterol intermediates of the mevalonate pathway regulate HMGCR degradation and SREBP-2 processing. J. Lipid Res. 2019, 60, 1765-1775. [CrossRef]

61. Tint, G.S.; Pan, L.; Shang, Q.; Sharpe, L.J.; Brown, A.J.; Li, M.; Yu, H. Desmosterol in brain is elevated because DHCR24 needs REST for Robust Expression but REST is poorly expressed. Dev. Neurosci. 2014, 36, 132-142. [CrossRef] [PubMed]

62. Russell, D.W. Cholesterol biosynthesis and metabolism. Cardiovasc. Drugs Ther. 1992, 6, 103-110. [CrossRef]

63. Lu, M.; Hu, X.H.; Li, Q.; Xiong, Y.; Hu, G.J.; Xu, J.J.; Zhao, X.N.; Wei, X.X.; Chang, C.C.; Liu, Y.K.; et al. A specific cholesterol metabolic pathway is established in a subset of HCCs for tumor growth. J. Mol. Cell Biol. 2013, 5, 404-415. [CrossRef]

64. Oh, T.G.; Wang, S.M.; Acharya, B.R.; Goode, J.M.; Graham, J.D.; Clarke, C.L.; Yap, A.S.; Muscat, G.E.O. The Nuclear Receptor, RORgamma, Regulates Pathways Necessary for Breast Cancer Metastasis. EBioMedicine 2016, 6, 59-72. [CrossRef]

65. Dooley, S.; ten Dijke, P. TGF-beta in progression of liver disease. Cell Tissue Res. 2012, 347, 245-256. [CrossRef]

66. Pelikan, R.C.; Iwata, J.; Suzuki, A.; Chai, Y.; Hacia, J.G. Identification of candidate downstream targets of TGFbeta signaling during palate development by genome-wide transcript profiling. J. Cell. Biochem. 2013, 114, 796-807. [CrossRef]

67. Matheu, A.; Collado, M.; Wise, C.; Manterola, L.; Cekaite, L.; Tye, A.J.; Canamero, M.; Bujanda, L.; Schedl, A.; Cheah, K.S.; et al. Oncogenicity of the developmental transcription factor Sox9. Cancer Res. 2012, 72, 1301-1315. [CrossRef]

68. Vizcaino, C.; Mansilla, S.; Portugal, J. Sp1 transcription factor: A long-standing target in cancer chemotherapy. Pharmacol. Ther. 2015, 152, 111-124. [CrossRef] [PubMed]

69. Vervoort, S.J.; Lourenco, A.R.; Tufegdzic Vidakovic, A.; Mocholi, E.; Sandoval, J.L.; Rueda, O.M.; Frederiks, C.; Pals, C.; Peeters, J.G.C.; Caldas, C.; et al. SOX4 can redirect TGF-beta-mediated SMAD3-transcriptional output in a context-dependent manner to promote tumorigenesis. Nucleic Acids Res. 2018, 46, 9578-9590. [CrossRef] [PubMed]

70. Caldwell, J.D.; Jirikowski, G.F. Sex hormone binding globulin and aging. Horm. Metab. Res. Horm. Stoffwechs. Horm. Metab. 2009, 41, 173-182. [CrossRef]

71. Hautanen, A. Synthesis and regulation of sex hormone-binding globulin in obesity. Int. J. Obes. Relat. Metab. Disord. J. Int. Assoc. Study Obes. 2000, 24 (Suppl. 2), S64-S70. [CrossRef]

72. Vaidya, D. Sex hormones and circulating lipoprotein levels. Future Lipidol. 2008, 3, 603-606. [CrossRef]

73. Cichoz-Lach, H.; Michalak, A. Oxidative stress as a crucial factor in liver diseases. World J. Gastroenterol. 2014, 20, 8082-8091. [CrossRef]

74. Wu, X.Z.; Chen, D. Origin of hepatocellular carcinoma: Role of stem cells. J. Gastroenterol. Hepatol. 2006, 21, 1093-1098. [CrossRef]

75. Fang, C.H.; Gong, J.Q.; Zhang, W. Function of oval cells in hepatocellular carcinoma in rats. World J. Gastroenterol. 2004, 10, 2482-2487. [CrossRef]

76. Acimovic, J.; Lovgren-Sandblom, A.; Monostory, K.; Rozman, D.; Golicnik, M.; Lutjohann, D.; Bjorkhem, I. Combined gas chromatographic/mass spectrometric analysis of cholesterol precursors and plant sterols in cultured cells. J. Chromatogr. B Anal. Technol. Biomed. Life Sci. 2009, 877, 2081-2086. [CrossRef]

77. Skubic, C.; Vovk, I.; Rozman, D.; Krizman, M. Simplified LC-MS Method for Analysis of Sterols in Biological Samples. Molecules 2020, 25, 4116. [CrossRef] [PubMed] 
78. Andersen, C.L.; Jensen, J.L.; Orntoft, T.F. Normalization of real-time quantitative reverse transcription-PCR data: A model-based variance estimation approach to identify genes suited for normalization, applied to bladder and colon cancer data sets. Cancer Res. 2004, 64, 5245-5250. [CrossRef]

79. Kosir, R.; Acimovic, J.; Golicnik, M.; Perse, M.; Majdic, G.; Fink, M.; Rozman, D. Determination of reference genes for circadian studies in different tissues and mouse strains. BMC Mol. Biol. 2010, 11, 60. [CrossRef]

80. Carvalho, B.S.; Irizarry, R.A. A framework for oligonucleotide microarray preprocessing. Bioinformatics 2010, 26, 2363-2367. [CrossRef]

81. Kauffmann, A.; Gentleman, R.; Huber, W. arrayQualityMetrics-A bioconductor package for quality assessment of microarray data. Bioinformatics 2009, 25, 415-416. [CrossRef]

82. Smyth, G.K. Linear models and empirical bayes methods for assessing differential expression in microarray experiments. Stat. Appl. Genet. Mol. Biol. 2004, 3. [CrossRef] [PubMed]

83. Matys, V.; Kel-Margoulis, O.V.; Fricke, E.; Liebich, I.; Land, S.; Barre-Dirrie, A.; Reuter, I.; Chekmenev, D.; Krull, M.; Hornischer, K.; et al. TRANSFAC and its module TRANSCompel: Transcriptional gene regulation in eukaryotes. Nucleic Acids Res. 2006, 34, D108-D110. [CrossRef]

84. Furge, K.; Dykema, K. PGSEA: Parametric Gene Set Enrichment Analysis, R package version 1.44 .0 ed; The R Foundation: Vienna, Austria, 2006.

Publisher's Note: MDPI stays neutral with regard to jurisdictional claims in published maps and institutional affiliations.

(C) 2020 by the authors. Licensee MDPI, Basel, Switzerland. This article is an open access article distributed under the terms and conditions of the Creative Commons Attribution (CC BY) license (http://creativecommons.org/licenses/by/4.0/). 